\title{
Microstructural Assessment of 316L Stainless Steel Using Infrared Thermography Based Measurement of Energy Dissipation Arising from Cyclic Loading
}

\author{
P.J. Seelan ${ }^{1}$, J.M. Dulieu-Barton ${ }^{2 a}$, F. Pierron ${ }^{1}$ \\ ${ }^{1}$ Department of Mechanical Engineering, School of Engineering, University of Southampton, \\ Highfield, Southampton, SO17 1BJ, UK \\ ${ }^{2}$ Department of Mechanical Engineering, School of Civil, Aerospace, and Mechanical Engineering, \\ University of Bristol, Queen's Building, Bristol, BS8 1TR, UK \\ ajanice.barton@bristol.ac.uk
}

\begin{abstract}
A procedure is developed that evaluates the energy dissipated from a material subject to cyclic loading and enables identification of the difference in material microstructure. It is demonstrated that the dissipated energy can be derived from specimens loaded in the elastic region using temperature measurements obtained by infrared thermography. To obtain accurate values of the small temperature changes resulting from the intrinsic dissipation below the yield point, a key part of the procedure is to eliminate the effect of external heat sources and sinks from the vicinity of the test specimen under investigation. To this end, a chamber was designed to minimise the external radiation whilst allowing the specimens to be cyclically loaded; the configuration of the chamber is described, alongside its integration into the procedure. A reference specimen was specifically introduced in the chamber to take into account the thermal exchanges between the specimen and the chamber environment. A data processing procedure, based on the thermomechanical heat diffusion equation, is applied to enable the dissipated energy to be derived from the temperature measurements. It is established that quantifying the amount of energy dissipation provides an opportunity to identify the material condition. The procedure is demonstrated on specimens made from 316L stainless steel containing a range of microstructures produced by different heat treatments. It is shown that the dissipative energy is dependent on the microstructure and that the dissipative source can be identified using the experimental procedure.
\end{abstract}




\section{Introduction}

It is well known that the mechanical deformation of a material is always accompanied by the dissipation of energy in the form of heat since the early works of Stromeyer [1] and Farren and Taylor [2]. Heat is released at the macroscopic scale because of irreversible thermodynamic processes occurring within the microstructure of the material [3]. It should be noted that the deformation does not have to be mechanically irreversible (i.e. loaded beyond the material macroscopic elastic region) to generate thermodynamic irreversibility. As the dissipated energy is released in the form of heat, a temperature change occurs, which could be measured using an infrared detector. Hence an opportunity arises to develop a non-contact tool to assess the material condition by virtue of determining the heat it dissipates under mechanical loading. In the case of elastic (mechanically reversible) loading, the intensity of the temperature change associated with the dissipated energy is small (a few $\mathrm{mK} \cdot \mathrm{s}^{-1}$ ) [4]. Hence, the temperature resolution of current commercially available IR cameras make it impossible to resolve the temperature change associated with the dissipated energy over a single load step directly from the IR camera output. Therefore, it is necessary to cyclically load the specimen at a reasonably high loading frequency enabling energy dissipation in every consecutive cycle. The high loading frequency also ensures that adiabatic conditions are achieved, so there is a gradual build-up of heat that gives rise to a temperature rise measurable by the infrared detector.

The temperature variation in a specimen undergoing elastic cyclic loading results (see Figure 1) from two different heat sources [5]: the thermoelastic heat source, $\theta_{\mathrm{TE}}$, and the dissipative heat source [6], $\theta_{\mathrm{D}}$. The thermoelastic source [7] causes a cyclic variation in temperature which has an amplitude proportional to the change in the sum of principal stresses. It has been used in over several decades as a stress analysis tool, e.g. [8-11], and more recently has been applied effectively in the field on inservice components [12].

Superimposed on the thermoelastic source is the dissipative heat source, which causes an increase in mean temperature of the material. It is dependent on the material microstructure and not the just the applied stress. The variation in temperature caused by the thermoelastic and the dissipative sources are shown in Figure 1. It is important to note that the intensity of the temperature change resulting from the thermoelastic effect is several orders of magnitude higher than the intensity of the dissipative temperature rise [13], for loadings below the macroscopic yield stress. The resulting temperature change is affected by thermal exchanges between the environment and the material [4], namely conduction, convection and radiation. It is therefore necessary to identify the dissipative heat source to obtain information that is dependent only on the material condition, as the temperature change is not an intrinsic quantity. It should be noted that when the heat source per cycle is considered the value 
will be the same for different frequencies (at least when these frequencies are comparable, barring strain rate dependence), although in the present paper the same loading frequency is used throughout the experimental work.

There are several microstructural phenomena responsible for dissipation, like viscous grain boundary sliding [14], atomic movements [15] and dislocation movements [16]. Mareau et al. [17] have shown that a micromechanical model based on dislocation motion could account for almost all of the heat dissipated during cyclic loading. It is noteworthy that the initiation of dislocation motion is governed by the Peierls-Nabarro (P-N) stress threshold $[18,19]$ and that dislocations start to move at stresses below macroscopic yield stress; this phenomenon is explored in the present paper. Various researchers have captured the temperature variations during a full fatigue test for the purpose of rapid fatigue life assessment. Although there have been successful attempts at obtaining fatigue limits purely from temperature rise that match previously known values of fatigue limits [20-22], a physical basis in which the microstructure can be linked to temperature evolutions has not yet been established, although some work in progress has been reported in [23, 24].

In the present paper the potential of dissipative heat source as tool for microstructural analysis is explored for the first time. Hence, the temperature increase, during the first few seconds of cyclic loading, is studied as opposed to a fatigue test until failure. In this case, the temperature increase is quite small and of the order of a few mK.s ${ }^{-1}$, which means several steps are required to accurately resolve the dissipative source. A reference specimen made out of the same material as the specimen is used to account for temperature exchanges between the environment following the method proposed in [4]. A specially designed chamber ensured that the heat sources from the surroundings were eliminated and is described in the 'Experimental Setup' section. The data processing sequence proposed in [25] is implemented to improve the resolution of the thermal measurements. The key aspect of the data processing procedure that enables this enhancement in thermal resolution is the spatial averaging process where every recorded infrared image frame of the surface temperature of the test specimen is spatially averaged. Therefore, a uniform distribution of the dissipative heat source and specimen the temperature is essential for obtaining a meaningful result with the spatial averaging. The detection threshold of the measurement is established, and it is demonstrated that the data processing procedure provides sufficient enhancement of the thermal resolution to allow the dissipative sources that occur prior to macroscopic yielding to be identified.

The material studied is 316L stainless steel which is widely used in many industries due to its resistance to corrosion, creep and high temperatures. It is also advantageous in the current work since it has a low thermal diffusivity which prevents heat generated from diffusing away quickly [26]. Additionally, Connesson et al. [27] have shown for 316L stainless steel that the dissipation occurs at low stress 
amplitude below the macroscopic yield stress [27]. A range of different microstructures of 316L stainless steel were generated through heat treatment; the heat treatment method and the resulting microstructure and mechanical properties are detailed in the 'Heat Treatment' section of the paper. The dissipative heat source was extracted from the experiments conducted on the heat treated specimens and it is established that the heat source is related to the microstructure and to dislocation density. The outcome of the work is understanding of the effect of microstructures on the dissipative heat source, which serves as a first step towards the use of dissipative heat source as tool for microstructural assessment.

\section{Experimental Setup}

The test-setup used 316L stainless steel strip specimens of $250 \mathrm{~mm}$ length by $20 \mathrm{~mm}$ width and $2 \mathrm{~mm}$ thickness. In all the tests described in the paper an Instron 8800 servo-hydraulic test machine was used to apply a cyclic load at different stress levels. In all the tests the loading frequency was $14 \mathrm{~Hz}$ and the stress ratio, $R_{s}$, was 0.1 . The specimen surface temperature was recorded using an infrared detector and the strains were obtained using a single strain gauge attached to the back surface of the specimen aligned longitudinally to the direction of the applied load. The infrared detector was a Flir SC 5000 model with the framerate set to $350 \mathrm{~Hz}$ with an integration time of $1200 \mu \mathrm{s}$. Prior to capturing the IR images an inbuilt non-uniformity correction was carried out using a high emissivity plate with a uniform temperature using the IR camera software inbuilt calibration. For most cases, the expected temperature rise due to cyclic loading is only a few $\mathrm{mK} \cdot \mathrm{s}^{-1}$. As metals generally have low emissivity, a thin layer of high emissivity coating (Electrolube matt black paint) was sprayed onto the specimens. The thickness of the paint coating was measured using a paint thickness gauge at different locations along the length of the specimen and was averaged. In all cases it was ensured that the thickness is in the range of 15-25 $\mu \mathrm{m}$, as recommended for thermoelastic stress analysis in [28].

With the application of the matt black paint, the emissivity of the specimens is only close to but not exactly unity. Hence, a minor portion of the temperature incorporated into the measurement consists of reflected radiation from external sources (i.e. sources not originating from the specimen). To account for the reflected radiation, a wooden chamber was specially designed to minimise the effect of external parasitic heat sources on the measurement. The chamber is shown mounted in the test machine in Figure 2 (a) with its component parts shown in Figure 2 (b). Once the specimen has been clamped in the grips, the two halves of the chamber are positioned on the stand and the chamber closed around the specimen. A recess feature was incorporated at the interconnecting surface between the two halves of the chamber, which eliminates any gaps (see Figure 2 (b)) that allow the passage of radiation into the chamber. One of the main sources of external radiation arises from the grips, so reflections from the grips are 
eliminated by designing the chamber so that the grips are outside the chamber. The wooden chamber is covered with aluminium foil to reflect external radiation. The effect of any remaining radiation is minimised by the wood which is a good heat insulator. The inner surface of the chamber was painted in black to minimise the effect of any small reflections within the chamber. The camera observes the inside of the chamber through an opening and the gap between the opening and the camera lens is sealed using a similar construction to that of the chamber, as shown in the actual setup in Figure 3 (a). Further to this, a black curtain enclosed the entire set up to further reduce any effect from heat sources in the surrounding laboratory.

A reference specimen (made of 316L stainless steel and also painted black) is introduced in the chamber to account for the effect of temperature fluctuations within the chamber, which include those caused by emitted and reflected radiation, and do not originate from the cyclic loading of the specimen. The reference specimen is mounted using masking tape (as shown in Figure 3 (b)) in the chamber so that it is adjacent to but does not touch the test specimen. The infrared camera field of view enables the temperature evolutions in the specimen and the reference specimen to be obtained simultaneously. The spatial average of the temperature of the reference specimen is subtracted from the spatial average of the temperature of the specimen to account for the changes in temperature that are not associated with the cyclic loading. The set-up means that all heat sources are eliminated apart from heat conducted through the test machine grips, which is eliminated by using an initial image before the cyclic loading is applied as described in the following section.

\section{Dissipative heat source from temperature measurements}

\subsection{The heat diffusion equation}

Assuming that the temperature is uniform through the thickness of the test specimen, a $2 \mathrm{D}$ form of the thermal energy balance at any point of the specimen is given as follows [4]:

$$
\begin{aligned}
& \rho C \frac{\partial T(x, y, t)}{\partial t}-k \nabla^{2} T(x, y, t)+\frac{2 B e T^{4}(x, y, t)}{d} \\
& +\frac{2 h}{d}\left(T(x, y, t)-T_{E}(x, y, t)\right)=S(x, y, t)+r_{S}(x, y, t)
\end{aligned}
$$

where $B$ is the Stefan-Boltzmann constant, $e$ is the emissivity, $k$ is the thermal conductivity of the material, $d$ is the thickness is the specimen, $h$ is the convective heat transfer coefficient, $T_{E}$ is the environmental temperature inside the chamber, $\nabla^{2} T$ is the 2D Laplacian of temperature which is given 
by $\frac{\partial^{2} T}{\partial x^{2}}+\frac{\partial^{2} T}{\partial y^{2}}, S$ is the thermomechanical source which is the sum of the thermoelastic source $S_{T E}$ and the dissipative heat source, $S_{D}$, and $r_{S}$ denotes all other external sources acting on the specimen, which includes radiation from the inner surface of the chamber, where the view factor for the radiation is considered to be 1 . Although the following derivation has been presented previously [4], it is important to represent again here to be able to link the data processing procedure to the derivation.

At time $t=0$, just before the cyclic loading is started, thermomechanical sources are not yet activated. Hence, the thermal energy balance per unit volume of the specimen at $t=0$ can be written as:

$$
\rho C \frac{\partial T(x, y, t)}{\partial t}_{(t=0)}-k \nabla^{2} T_{0}(x, y)+\frac{2 B e T_{0}^{4}(x, y)}{d}+\frac{2 h}{d}\left(T_{0}(x, y)-T_{E 0}(x, y)\right)=r_{S 0}(x, y)
$$

where the subscript zero denotes values at time $t=0$ and $\frac{\partial T(x, y, t)}{\partial t}$ it=0) is the gradient of the temperature with respect to time at $t=0$.

By subtracting an initial infrared image at $t=0$, it is possible to eliminate the initial temperature gradient caused by the heat conduction from the test machine grips connected to the actuator by subtracting Equation (2) from Equation (1):

$$
\begin{aligned}
& \rho C\left[\frac{\partial \theta_{S}(x, y, t)}{\partial t}-\frac{\partial \theta_{S}(x, y, t)}{\partial t}{ }_{(t=0)}\right]-k \nabla^{2} \theta_{S}(x, y, t)+\frac{2 B e}{d}\left(T^{4}(x, y, t)-T_{0}^{4}(x, y)\right) \\
& +\frac{2 h}{d}\left(T(x, y, t)-T_{0}(x, y)\right)-\frac{2 h}{d}\left(T_{E}(x, y, t)-T_{E 0}(x, y)\right)=S(x, y, t)+r_{S}(x, y, t)-r_{S 0}(x, y)
\end{aligned}
$$

where $\theta_{S}(x, y, t)=T(x, y, t)-T_{0}(x, y)$.

As the temperature variations are small hence $T$ recorded at each infrared image frame and $T_{0}$ are very close. By assuming that $T \approx T_{0}$, the radiation term can be linearised, which results in a single time constant describing the radiation and convective heat transfer denoted as $\tau$ [29]. Equation (3) then becomes: 


$$
\begin{aligned}
& \rho C\left[\frac{\partial \theta_{S}(x, y, t)}{\partial t}-\frac{\partial \theta_{S}(x, y, t)}{\partial t}\right]-k \nabla^{2} \theta_{S}(x, y, t)+\rho C \frac{\theta_{S}(x, y, t)}{\tau}-\frac{2 h}{d}\left(T_{E}(x, y, t)-T_{E 0}(x, y)\right) \\
& =S(x, y, t)+r_{S}(x, y, t)-r_{S 0}(x, y)
\end{aligned}
$$

By taking the average temperature in the $\mathrm{x}$ and y direction (see Figure 3 (b)), the spatially averaged version of Equation (4) is obtained:

$$
\rho C\left[\frac{\partial \overline{\bar{\theta}}_{S}(t)}{\partial t}-\frac{\partial \overline{\bar{\theta}}_{S}(t)}{\partial t}{ }_{(t=0)}\right]-\frac{k}{l} \int_{-l / 2}^{l / 2} \nabla^{2} \bar{\theta}_{S}(y, t) d y+\rho C \frac{\overline{\bar{\theta}}_{S}(t)}{\tau}-\frac{2 h}{d}\left(\overline{\bar{T}}_{E}(t)-\overline{\bar{T}}_{E 0}\right)=\overline{\bar{S}}(t)+\overline{\bar{r}}_{S}(t)-\overline{\bar{r}}_{S 0}
$$

where the single bar represents the mean in the $\mathrm{x}$-direction and the double bar represents spatial mean in both $\mathrm{x}$ and $\mathrm{y}$ directions. As heat conduction is small in the horizontal (or $x$ ) direction, but in the vertical (or $y$ ) direction is relatively large, the effect of heat conduction is accounted for by evaluating the Laplacian by spatially averaging in the $x$-direction only.

Similarly, the spatially averaged heat diffusion equation of the reference specimen is as follows:

$$
\rho C\left[\frac{\partial \overline{\bar{\theta}}_{R}(t)}{\partial t}-\frac{\partial \overline{\bar{\theta}}_{R}(t)}{\partial t}(t=0)\right]+\rho C \frac{\overline{\bar{\theta}}_{R}(t)}{\tau}-\frac{2 h}{d}\left(\overline{\bar{T}}_{E R}(t)-\overline{\bar{T}}_{E R 0}\right)=\overline{\bar{r}}_{R}(t)-\overline{\bar{r}}_{R 0}
$$

where the subscript $R$ denotes reference specimen. Note, as the reference specimen is not cyclically loaded there is no source term, and no conduction as the specimen is held with an insulating material, so, the Laplacian term is not present.

The spatial averaging procedure assumes that the spatial mean of the thermomechanical sources is representative of the local thermomechanical sources. While this is limited to homogeneous specimens under uniform stress states, it offers the advantage of noise reduction in proportion to the square root of the number of measurement points. Assuming that the spatially averaged environmental temperature variation is the same on the specimen and the reference specimen (i.e. $\overline{\bar{T}}_{E}(t)-\overline{\bar{T}}_{E 0} \approx \overline{\bar{T}}_{E R}(t)-\overline{\bar{T}}_{E R 0}$ ) and the effect of the radiative emission and reflection inside the chamber has the same effect on the specimen and the reference specimen $\left(\overline{\bar{r}}_{S}(t)-\overline{\bar{r}}_{S 0} \approx \overline{\bar{r}}_{R}(t)-\overline{\bar{r}}_{R 0}\right)$ subtracting Equation (6) from Equation (5) gives: 


$$
\rho C\left[\frac{\partial \overline{\bar{\theta}}(t)}{\partial t}-\left(\frac{\partial \overline{\bar{\theta}}(t)}{\partial t}\right)_{(t=0)}\right]-\frac{k}{l} \int_{-l / 2}^{l / 2} \nabla^{2} \bar{\theta}(y, t) d y+\rho C \frac{\overline{\bar{\theta}}(t)}{\tau}=\overline{\bar{S}}(t)
$$

where $\overline{\bar{\theta}}(t)$ is the overall temperature difference between the spatial average of the specimen temperature variation and the reference specimen temperature variation given by $\overline{\bar{\theta}}(t)=\overline{\bar{\theta}}_{S}(t)-\overline{\bar{\theta}}_{R}(t)$.

$\overline{\bar{\theta}}(t)$ consists of the thermoelastic temperature variation, $\overline{\bar{\theta}}_{T E}$, and dissipative temperature rise, $\overline{\bar{\theta}}_{D}(t)$, so the following expression can be applied:

$$
\overline{\bar{\theta}}(t)=\overline{\bar{\theta}}_{T E}(t)+\overline{\bar{\theta}}_{D}(t)
$$

Therefore, $\overline{\bar{\theta}}_{T E}$ can be subtracted from the overall temperature change to give only the dissipative heat rise and hence the dissipative heat source is identified as follows:

$$
\rho C\left[\frac{\partial \overline{\bar{\theta}}_{D}(t)}{\partial t}-\left(\frac{\partial \overline{\bar{\theta}}_{D}(t)}{\partial t}\right)_{(t=0)}\right]-\frac{k}{l} \int_{-\frac{l}{2}}^{\frac{l}{2}} \nabla^{2} \bar{\theta}_{D}(y, t) d y+\rho C \frac{\overline{\bar{\theta}}_{D}(t)}{\tau}=\overline{\bar{S}}_{D}(t)
$$

\subsection{Data processing procedure}

The data processing procedure was implemented in MATLAB (the script will be made available in a data file upon publication). Figure 4 (a) shows a representation of the temperature data obtained from the specimen and the reference specimen using the infrared detector. To remove the temperature variation caused by the grips in the specimen and any other fixed pattern noise such as the reflection of the cold detector in both the specimen and the reference specimen, the first image (before the start of the cyclic loading) is subtracted from the subsequent images as shown in Figure 4 (a). The data is now in the form given by Equation (3). Next spatial averaging is carried out to provide the temperature data in the form of Equation (5) and Equation (6) for the test specimen and the reference specimen respectively. The influence of the environmental temperature fluctuation in the chamber is then eliminated by subtracting the spatial mean of the reference specimen temperature variation from the spatial mean of the specimen temperature variation, as given by Equation (7). 
The resulting overall temperature variation is shown in Figure 4 (b), which is represented by Equation (8), i.e. the sum of the thermoelastic temperature variation and the mean temperature rise. The small variation in the temperature at the start of the cyclic loading is due to the test machine settling. The next step is to remove the thermoelastic temperature variation from the overall temperature variation. The thermoelastic temperature variation for a specimen loaded in uniaxial tension is proportional to the range of the cyclic loading, so it can be calculated from the known applied load. It is possible to record the load signal in Digital Level (DL) using the infrared camera signal input port so evaluating the constant of proportionality between the load signals in DL and the temperature variation directly means that the thermoelastic constant does not have to be evaluated (i.e. the ratio of the amplitudes of the load signal in DL and temperature in K as detailed in [30]). A small phase difference and hence time delay exists between the recorded load signal and the thermoelastic response, as a result of the camera electronics. This is corrected by shifting the load signal by the time delay using interpolation, hence an in-phase thermoelastic temperature variation is obtained. The mean temperature rise is determined by subtracting the thermoelastic temperature variation from the overall temperature signal to give data in the form of Equation (9).

The noise contained in the mean temperature rise resulting from the subtraction is significant, because further data processing is required to obtain the dissipative source using Equation (9), which means differentiating the mean temperature rise, i.e. an operation that will amplify noise. So it is necessary to smooth the mean temperature rise with a moving average window the size of three loading cycles (75 data points) as suggested in [25] where it was shown that this kernel size maintained the detail while reducing the noise. This smoothing operation was applied three times consecutively and the resulting noise reduction is shown in Figure 5.

The first term in Equation (9) is the heat rate term, $\rho C\left[\frac{\partial \overline{\bar{\theta}}_{D}(t)}{\partial t}-\left(\frac{\partial \overline{\bar{\theta}}_{D}}{\partial t}\right)_{(t=0)}\right]$, which accounts for any initial gradient in the specimen prior to cyclic loading as the gradient of temperature rise at $t=0$ is subtracted from the gradient of the dissipative temperature rise. It should be noted that the evaluation of $\left(\frac{\partial \overline{\bar{\theta}}_{D}}{\partial t}\right)_{(t=0)}$ is not possible due to the discontinuity in the temperature gradient at $t=0$. As such, the initial gradient was evaluated just before the start of the cyclic loading i.e. $\left(\frac{\partial \overline{\bar{\theta}}_{D}}{\partial t}\right)_{\left(t=0^{-}\right)}$where $t=0^{-}$ indicates the time just before the start of the cyclic loading. The gradient of the temperature rise was obtained by numerical differentiation using a finite difference approximation. The initial temperature gradient was obtained using a linear least square fit of the temperature variation over five seconds before 
the start of cyclic loading which is an accurate means of capturing any trends in initial temperature variation compared to evaluating the gradient just at $t=0$.

The second term in Equation (9) is a conduction term, which is obtained from a least square approximation of the vertical profile of the temperature of the specimen using a second order polynomial. The resulting second order coefficient for each vertical temperature profile gives the Laplacian term, which is a measure of the amount of curvature in the vertical temperature profile. Figure 6 shows that initially, there is no temperature gradient in the vertical direction as the temperature difference due to conduction from the lower grip being eliminated by the image subtraction procedure. As the test progresses the conduction is accounted for by the Laplacian, the increase in conduction at the grips shown in Figure 6 is because the specimen is in contact with the grips. Usually, the heat conduction in the case of elastic cyclic loading is minimal (i.e. less than $10 \%$ of the heat rate term) but becomes more significant in the presence of plasticity.

The linearised convective and radiative heat transfer time constant, $\tau$, used in the third term in Equation (9) and required to calculate the radiative and convective heat transfer term was obtained by heating the reference specimen to approximately $50{ }^{\circ} \mathrm{C}$ using a heat gun. The temperature of the reference specimen was measured using the infrared detector with a frame rate of $5 \mathrm{~Hz}$ with the same integration time as that of the test for $400 \mathrm{~s}$, as the specimen cooled. The resulting cooling curve was fitted using an exponential function, so that the time constant could be determined as the coefficient of the exponential decay function. This time constant depends on the specimen material as well as the environmental temperature. While 316L stainless steel is used throughout this work, the experiments were conducted at different times of the year and hence different room temperatures, so the time constant was evaluated once on every day the experiments were performed. The time constant typically ranges from $180 \mathrm{~s}$ to $220 \mathrm{~s}$ for the experiments conducted.

\subsection{Establishing the detection threshold}

The detection threshold was determined by recording data without applying the cyclic load. In this case, the dissipative heat source is not activated so any temperature variation is caused by the detector noise or thermal exchanges with the surroundings. In the experimental set-up it is assumed that both the specimen and the reference specimen experience the same thermal exchanges with the surroundings, so in theory by subtracting one from the other the temperature variation should be zero. In Figure 7 (a) temperature values obtained from the experimental set-up are shown and indicates the calculation $\overline{\bar{\theta}}(t)=\overline{\bar{\theta}}_{S}(t)-\overline{\bar{\theta}}_{R}(t)$. Both $\overline{\bar{\theta}}_{S}$ and $\overline{\bar{\theta}}_{R}$ contain significant systematic noise due to the chamber environment. It is clear that the extracted value, $\overline{\bar{\theta}}$, which is used in the subsequent calculations to determine the dissipative source, is very close to zero and that the systematic noise due to the 
environment in the chamber has been reduced. In Figure 7 (b) the temperature variations are processed into the dissipative source using Equation (9). The means and the standard deviations of the resulting heat source was calculated for the 10 sets of data collected and averaged in time to provide a single value. The black line in Figure 7 (b) shows the mean of the heat sources to be of $0.05 \mathrm{mK} . \mathrm{s}^{-1}$, which is the uncertainty associated with the temporal mean of a stabilized heat source. Twice the standard deviation provides the detection threshold, above which all measurements can be assumed significant with a confidence level of 95\%, indicated by the red lines in Figure 7 (b). Due the random nature of the detector noise, the computed heat source is also random and hence positive and negative thresholds are generated. When divided by the density and specific heat capacity at constant pressure, a detection threshold is obtained in terms of heat rate, here $0.72 \mathrm{mK} . \mathrm{s}^{-1}$. This implies that, in a period of one second, the combination of the thermographic measurement and data processing method has a noise equivalent of $0.72 \mathrm{mK}$. The measurement is well below the sensor NETD and is achieved at the cost of losing any local information, because of the temperature averaging and smoothing. As the specimens used in the present work (see Section 4) are macroscopically homogeneous samples the spatial averaging approach is acceptable.

\section{Creating the microstructural variations in 316L stainless steel}

The 316L material in the as received condition is cold rolled and has a fine grain structure, so the aim is to produce different grain sizes through heat treatment, hence the grain size can only be made larger than that of the as received condition. Further cold work of the material might produce finer grains but the only option was heat treatment. As previous work [25, 27] has shown the dissipation is related to dislocation density, the target is to use the heat treatment to achieve a range of dislocation conditions. Two mechanisms exist when metallic materials are annealed. The first is called recovery, which occurs at lower temperatures, where dislocations with opposite signs are annihilated and subsequently rearranged into a lower energy configuration [31]. This mechanism is responsible for a minimal reduction in dislocation density leading to only a partial residual stress relief [32]. The second mechanism occurs when the material is exposed to temperatures exceeding the recrystallisation temperature, new dislocation-free grains are formed, replacing old ones. The recrystallized specimens have a dislocation density that is much lower than the original cold-rolled ones, resulting in complete residual stress relief [32].

The heat treatments were carried out in a tube furnace connected to a vacuum pump, on test specimens that were cut to size. The vacuum was essential in preventing the surface from oxidising as oxide layers can create a thermal insulating layer that will hinder the infrared imaging. From the elevated temperature the specimens were control-cooled to room temperature so as to avoid shape distortions in such thin specimens. The grain size generated in the heat treated specimens was identified from optical 
micrographs using the intercept procedure as given in ASTM-E112-13. As the material yield stress is particularly sensitive to heat treatment and grain size, mechanical properties of the specimens were obtained in monotonic tensile tests. Rectangular rosette strain gauges $(0 / 45 / 90)$ were used for strain measurement to ensure that accurate mechanical properties could be obtained, unaffected by strain gauge misalignment. The strain gauge rosettes were attached to the front and back surface of the specimen. The specimens were loaded in displacement control at a strain rate of $0.0005 \mathrm{~s}^{-1}$ using a servohydraulic test machine. By averaging the front and back strains, the stress-strain curves were then plotted. As some of the stress-strain curves do not have a well-defined yield stress, the conventional $0.2 \%$ offset yield stress was recorded consistently. Young's modulus was found by a least square fit of the points between $0.1 \%$ and $0.4 \%$ of the estimated yield stress. Subsequently, the $0.2 \%$ offset yield stress was found from the stress strain curves. Additionally, Vickers microhardness values were measured under $50 \mathrm{~g}$ load with $10 \mathrm{~s}$ dwell time using Matsuzawa Seiki MHT - 1 micro hardness tester. The average and standard deviation of the hardness values of 10 indents per specimen were calculated.

The exact temperature to attain different dislocation states are difficult to determine because it is strongly dependent on the initial state. As shown by Donadille et al. [33], 316L stainless steel can recrystallize at a temperature as low as $800{ }^{\circ} \mathrm{C}$ when subjected to $40 \%$ cold working, whereas at a lower level of cold work, e.g. 10\%, a higher recrystallization temperature is required. Due to the variability in the cold rolling process [34] used to produce the material, the exact extent of the previous deformation is not known. Hence, the temperature and dwell time of the heat treatment performed were tailored using microscopic evaluation of trial test pieces.

Micrographs of the specimen microstructure are shown in Figure 8 (a) - (e), stress-strain curves in Figure 9 (a) - (c) and a summary of the hardness values and $0.2 \%$ offset yield stress is given in Table 1 . In addition to the microstructure in the as received condition (specimen A - Figure 8 (a)), two specimens subjected to recovery and one specimen each subjected to recrystallisation and grain growth were produced. Temperatures of $450{ }^{\circ} \mathrm{C}$ and $650{ }^{\circ} \mathrm{C}$ (specimens B and C respectively Figure 8 (b) and (c)) with a dwell time of one hour in each case were used for the recovery process. As expected, the grain sizes were similar to the as received specimens. While the recovery process does not alter the $0.2 \%$ conventional yield stress nor the hardness, it was found from the stress-strain curves (see Figure 9 (a)) that the transition between elastic and plastic behaviour becomes more apparent at higher annealing temperatures.

In the preliminary stages of the work [35], it was found that significant grain growth occurred at a temperature of $1050^{\circ} \mathrm{C}$ in just 2 minutes of dwell time. This implies that recrystallisation could take place without significant grain growth at a lower temperature, i.e. $950{ }^{\circ} \mathrm{C}$ at a dwell time of 30 minutes (specimen D). As can be seen in the micrograph (Figure 8 (d)) no change in grain size has occurred but 
the stress strain curve of the monotonic tensile test shows that it has a very sharp transition from elastic to plastic behaviour suggesting recrystallisation has occurred (see Figure 9 (b)). Grain growth was induced by elevating the target temperature further to $1050{ }^{\circ} \mathrm{C}$ (specimen $\mathrm{E}$ ). At this temperature the grain size has more than doubled (as shown in Figure 8 (e)). Consistently the yield stress has reduced as shown in Figure 9 (c). The heat treatment procedure along with the mechanical properties of the different specimens are summarised in Table 1 . As seen in Table 1, the hardness also reduced significantly from the as received to the recrystallized states and reduced even further in the specimen in which grain growth had occurred.

\section{Effect of microstructure on the dissipative heat source}

\subsection{Application of load to generate heat source}

A sequence of cyclic loading was applied to specimens A - E (as defined in Table 1) where the applied maximum stress level in the cycle was a fraction of the respective yield stress defined in Table 1. For all the specimens, stresses were applied at loading frequency of $14 \mathrm{~Hz}$ with a stress ratio $\mathrm{R}_{\sigma}$ of 0.1 . In the case of the as received specimen, the fractions of yield stress are indicated on the stress strain curve shown in Figure 10. The same fractions of yield stress were used for all specimens for the maximum applied stress in the load cycle. Using the same fractions of yield stress allows for the energy dissipated to be compared for each microstructure. The sequence of the applied loading is provided in Table 2 . The specimens are subjected to each stress level for approximately 300 load cycles (approximately 20 s) under load control before increasing the applied stress to next level in the sequence given in Table 2. Some sample data from the experiments will be made available in a data file upon publication.

\subsection{Elastic cyclic loading}

Under cyclic loading below macroscopic yield stress, it is typical to expect a linear temperature rise as shown in Figure 11 (a). The heat source is predominantly made up of the heat rate term while the conduction term and convection and radiation term are usually very small. As such, the heat source variation is well approximated by the heat rate, as shown in Figure 11 (b), as the stable heat source indicates viscoelastic behaviour or reversible movement of anchored dislocations [25]. At this loading level, heat sources are generally very small and close to the detection threshold, as also shown in Figure 11 (b). The area under a hysteresis loop of the stress-strain curve corresponds to the energy dissipated [3, 36]. However, there are complications involved in obtaining a meaningful estimate of the energy dissipated from the hysteresis loop in the case of elastic loading, particularly for metals, as detailed in [25]. It is important to note that strain gauge excitation did not cause any significant localised heating during the experiment, as the duration of each test was only $15 \mathrm{~s}$, so that the initial image 
subtraction was sufficient to account for any small deviations in temperature caused by the operation of the strain gauge.

When the cyclic loading was fully elastic (steps 1-6 in Table 2), the heat source was constant over time as demonstrated earlier. As such, the temporal mean of the evolution of the spatially averaged heat source can be used. The effect of the temporal mean is shown in Figure 12 where the spatially averaged heat source of two different tests on the same specimen under fully elastic loading (see Figure 12 (a)) is temporally averaged resulting in Figure 12 (b). Note that the load level in Test 2 is higher than the load level in Test 1 . As mentioned in the detection threshold section, the uncertainty or error bar in each case is $0.05 \mathrm{mK} \cdot \mathrm{s}^{-1}$. Temporally averaging also aids comparison of results between the different specimens.

It is important that the variation of the heat source due to the variability in the material in the as received condition is known. Any variability would superimpose on the dissipation level of other microstructures, as observed by Connesson et al. [27] where inconsistencies in the heat source occurred in the same batch of material. Hence, the repeatability is assessed by subjecting two as received specimens from the same batch of material to the same set of loading levels. Figure 13 shows that at all loading levels with the exception of the highest stress level, the derived dissipative sources for each specimen are very close, within the error, demonstrating excellent repeatability. The discrepancy at the highest stress level maybe attributed to the test machine load control parameters. The very close agreement of the results from the two specimens in the as received condition meant that it was unnecessary to conduct similar repeatability test on the heat treated specimens as the base material for the heat treated specimens came from the same billet of material. It should be noted that the specimens were made from a sheet metal of a relatively small area. The repeatability of the procedure was also confirmed in [25, 27].

Figure 14 shows that the energy dissipated generally increases as the stress level is increased for all the different microstructures. This is because at higher stress level the mobility of the dislocations is higher. With the highest dislocation density, the energy dissipated by the as received specimen is the highest across all loading levels as expected. The specimens that have been subjected to recovery dissipate less energy than the specimens made from the as received material due to a minor reduction in dislocation density whereas the dissipation in the specimens that have undergone recrystallization and grain growth is not at a measurable level except at the highest stress level close to the yield stress. However, when comparing the energy dissipated by the as received and the $450{ }^{\circ} \mathrm{C}$ heat treated specimens, the dissipation is generally greater in the as received specimen except at the highest stress level. At the loading level where the maximum stress is the yield stress, both the monotonic tensile test curve for the $450{ }^{\circ} \mathrm{C}$ and the as received specimens show a similar gradual transition from elastic plastic behaviour 
(see Figure 9 (a)), whereas at heat treatments of $650^{\circ} \mathrm{C}$ and above there is a sharp transition from elastic to plastic behaviour thus providing an explanation for their dissipation being the same at the highest stress level.

\subsection{Elastic-plastic cyclic loading}

Under elastic-plastic cyclic loading where a part of the applied stress is above the initial yield stress, a temperature increase such as in Figure 15 (a) is obtained. After an initial rapid rise in temperature (as noted in [37]), it becomes linear again. This is more evident when represented as a heat source (see Figure 15 (b)), where the rapid temperature rise is shown as the large peak in the heat source and as temperature rise becomes linear, the heat source stabilises. In terms of microstructural evolution during this loading, the peak in the heat source is caused by the increase in thermodynamic irreversibility due the creation of new dislocations as plasticity occurs as explained in [6]. As the material hardens, the heat source gradually stabilises - this accommodation signifies the drop in the contribution of plasticity to the energy dissipated and the eventual return to fully viscoelastic behaviour [6].

As the applied stress is increased beyond the macroscopic yield stress, strain hardening occurs. An example of the cyclic stress strain curve is given in Figure 15 (c). Although, it is difficult to discern the incremental stress-strain curves the strain hardening process is evident, and is also noted in [38] for displacement controlled tests on 316L stainless steel. However, under load control, the applied stress remains constant, whereas the strain increases at every cycle due to the accumulation of plastic strain. Even though the loop is open, an approximate plastic work per cycle is still obtainable from the stressstrain data. Here, the plastic work obtained for the hysteresis loop is compared to the energy dissipated for the specimens with different dislocation microstructures.

An approximate plastic work in a cycle could be obtained from the stress and strain data as follows [25]:

$$
W_{P}^{i}=\int_{t_{i}}^{t_{i}+t_{p}} \sigma \dot{\varepsilon}_{p} d t \approx \sigma_{\max } \Delta \varepsilon_{p}^{i}
$$

where $W_{P}^{i}$ the plastic work in a cycle, $i$ is the cycle number, $t_{i}$ is the time at the start of every cycle, $t_{p}$ is the duration or period of every cycle, $\sigma_{\max }^{i}$ and $\Delta \varepsilon_{P}^{i}$ are the maximum stress and incremental plastic strain for that cycle respectively. To be able to compare the plastic work in a cycle to energy dissipated, the energy dissipated per cycle has to be first defined: 


$$
E_{D}^{i}=\int_{t_{i}}^{t_{i}+t_{p}} S_{D} d t
$$

where $E_{D}^{i}$ is the energy dissipated per cycle in units of $\mathrm{J}^{-\mathrm{m}^{-3}}$.cycle $\mathrm{c}^{-1}$. This then enables the comparison of plastic work and the energy dissipated (see Figure 16). In comparing these quantities, it is important to be aware of the wider context of energy balance [25]:

$$
W_{M}^{i}=W_{V E}^{i}+W_{P}^{i}=E_{D}^{i}+E_{S}^{i}
$$

where $W_{M}^{i}$ is the input mechanical work, $W_{V E}^{i}$ is the viscoelastic work, $W_{P}^{i}$ is the plastic work and $E_{S}^{i}$ is the stored energy.

The stored energy in equation (12) refers to the energy used to modify the material microstructure, i.e. the energy used to create new dislocations. It follows that this term is only present when there is plasticity. It could be said that the plastic work at the start of the test and at the end of the test is very small (see Figure 16). As such, the amount of energy that is stored is also small. Hence, the energy dissipated at these instances could be attributed to purely viscoelastic work. This then implies that the final viscoelastic work is much higher compared to the initial viscoelastic work. This increase reflects the fact that the dislocation density has increased from its initial level due to plastic straining.

Cyclic loading is applied with a maximum stress of approximately $1.2 \sigma_{y}$ (step 7 in Table 2) for specimens A - E to achieve this cyclic hardening. However, the strain data was only recorded for specimens A, D and E. The comparison of energy dissipation per cycle and its corresponding plastic work during strain hardening is shown in Figure 16, Figure 17 (a), and (b) for the as received (specimen A), grain growth (specimen E), and recrystallized (specimen D) specimens respectively. Plastic work in all the three cases is always lower than the energy dissipated due to the significant contribution from viscoelastic work. The difference between the plastic work and the total dissipated energy increases as strain hardening occurs. This is because as the strain hardening progresses, the dislocation density increases and therefore the viscoelastic work also increases bringing the energy dissipated to a level much higher than the plastic work. The initial difference between the energy dissipated and plastic work is not noticeable for the grain growth (specimen E - Figure 17 (a)) and recrystallized (specimen D - Figure 17 (b)), as it could be seen from its corresponding fully elastic cyclic loading results that the dissipation in those cases were generally low. This is not the case for the as received specimen (specimen A - Figure 16) as there is a noticeable difference in the two quantities at the start of cyclic loading. This is because of the higher initial dislocation density in the as received 
specimen compared to the other two specimens that then causes a significantly higher viscoelastic dissipation.

\subsection{Post-yielding behaviour}

Finally, subsequent to hardening, the lower levels of stress were applied on the specimens to observe the effect of plastic straining (steps $8-13$ in Table 2). Once the specimen has yielded, fully elastic loading at the same stress level as in the previous case was applied. It was found that all the specimens dissipated more due to the increase in dislocation density through plastic straining (see Figure 18); this is consistent with the findings of Connesson et al.[27]. The results demonstrate that the sensitivity of the energy dissipation is such that even small changes in the microstructure due to plastic deformation can be detected.

\section{Conclusions}

It was successfully demonstrated with a combination of suitable experimental setup and data processing that the energy dissipation response of different microstructures of 316L obtained through heat treatment was significantly different under fully elastic cyclic loading. The role of the experimental setup which includes the novel chamber and the reference specimen as well as the data processing procedure was paramount in reducing the detection threshold to a level where energy dissipation from stresses below the yield stress was measurable. The sensitivity of the energy dissipation tests was demonstrated using microstructures that were barely distinguishable in micrographs. The dissipative source from five different heat treatments was clearly different, establishing that the approach has clear benefits over traditional inspections for determining the condition of a material. The repeatability of the approach was also established, albeit on only two practically identical specimens, confirming the findings of previous work [4, 6, 25,27], and further demonstrating the applicability of the approach.

The methodology for establishing the dissipative source can be considered to be non-destructive in nature as a measurable change in the dissipative source was established prior to the material yielding. Hence, the technique has the potential to provide complementary material information after which it can be subjected to other destructive characterisation techniques. Furthermore, the post-yield dissipative heat source was also found to be very sensitive to the cyclic hardening process.

More importantly, the results are encouraging in that it shows that the energy dissipation could be extended to assess more complex heterogeneous microstructures like that of a weld non-destructively. However, as the material microstructure and condition in the proximity of a weld is non-uniform, a suitable image processing procedure must be devised that provides the necessary thermal resolution 
without the need to conduct the spatial averaging used in the present paper; this is the focus of current work.

\section{Data Provision}

Data supporting this study are openly available from the University of Southampton repository at https ://doi.org/xxx.

\section{Acknowledgements}

The work described in the paper was part of an Innovate UK sponsored collaborative project under the "Developing the civil nuclear power supply chain” call, number 101438, Residual Stress and Structural Integrity Studies using Thermography (RESIST). Project partners include Enabling Process Technologies Ltd, University of Southampton, National Physical Laboratory, TWI, EDF Energy and Wood Plc. The experimental work described in the paper was conducted in the Testing and Structures Research Laboratory (TSRL) at the University of Southampton https://www.southampton.ac.uk/engineering/research/facilities/360/tsrl_360.page. The authors acknowledge the support received from Dr Andy Robinson, the TSRL Principal Experimental Officer. 


\section{References}

[1] C. Stromeyer, "The determination of fatigue limits under alternating stress conditions", Proc. R. Soc. Lond. A, vol. 90, no. 620, pp. 411-425, 1914.

[2] W. Farren and G. I. Taylor, "The heat developed during plastic extension of metals", Proceedings of the Royal Society of London. Series A, Containing Papers of a Mathematical and Physical Character, vol. 107, no. 743, pp. 422-451, 1925.

[3] A. Chrysochoos, "Infrared thermography applied to the analysis of material behavior: a brief overview", Quantitative InfraRed Thermography Journal, vol. 9, no. 2, pp. 193-208, 2012, doi: 10.1080/17686733.2012.746069.

[4] F. Maquin and F. Pierron, "Refined experimental methodology for assessing the heat dissipated in cyclically loaded materials at low stress levels", Comptes Rendus Mécanique, vol. 335, no. 3, pp. 168-174, 2007, doi: 10.1016/j.crme.2007.02.004.

[5] A. Chrysochoos and J. C. Dupre, "An infrared set-up for continuum thermomechanics", presented at the QIRT, Château-Malabry, France, 1992.

[6] F. Maquin and F. Pierron, "Heat dissipation measurements in low stress cyclic loading of metallic materials: from internal friction to micro-plasticity", Mechanics of Materials, vol. 41, no. 8, pp. 928-942, 2009, doi: 10.1016/j.mechmat.2009.03.003.

[7] J. M. Dulieu-Barton, "Thermoelastic stress analysis", in Optical Methods for Solid Mechanics: A Full-Field Approach, First ed. New York: John Wiley \& Sons, 2012, pp. 345-366.

[8] A. L. Gyekenyesi, "Thermoelastic stress analysis: an NDE tool for residual stress assessment of metallic alloys", Journal of Engineering for Gas Turbines and Power, vol. 124, no. 2, p. 383, 2002, doi: 10.1115/1.1417982.

[9] J. Dulieu-Barton and P. Stanley, "Development and applications of thermoelastic stress analysis", The Journal of Strain Analysis for Engineering Design, vol. 33, no. 2, pp. 93-104, 1998.

[10] L. Baker and J. Webber, "Thermoelastic stress analysis", Journal of Modern Optics, vol. 29, no. 4, pp. 555-563, 1982.

[11] N. Rajic and D. Rowlands, "Thermoelastic stress analysis with a compact low-cost microbolometer system", Quantitative InfraRed Thermography Journal, vol. 10, no. 2, pp. 135158, 2013.

[12] R. C. Tighe, G. P. Howell, J. P. Tyler, S. Lormor, and J. M. Dulieu-Barton, "Stress based nondestructive evaluation using thermographic approaches: from laboratory trials to on-site assessment", NDT \& E International, vol. 84, pp. 76-88, 2016, doi: 10.1016/j.ndteint.2016.08.005.

[13] B. Berthel, A. Chrysochoos, B. Wattrisse, and A. Galtier, "Infrared image processing for the calorimetric analysis of fatigue phenomena", Experimental Mechanics, vol. 48, no. 1, pp. 7990, 2007, doi: 10.1007/s11340-007-9092-2.

[14] T. i.-S. Kê, "Experimental evidence of the viscous behavior of grain boundaries in metals", Physical Review, vol. 71, no. 8, pp. 533-546, 1947, doi: 10.1103/PhysRev.71.533.

[15] J. Snoek, "Effect of small quantities of carbon and nitrogen on the elastic and plastic properties of iron", Physica, vol. 8, no. 7, pp. 711-733, 1941.

[16] A. Granato and K. Lücke, "Theory of mechanical damping due to dislocations", Journal of Applied Physics, vol. 27, no. 6, p. 583, 1956, doi: 10.1063/1.1722436. 
[17] C. Mareau, V. Favier, B. Weber, A. Galtier, and M. Berveiller, "Micromechanical modeling of the interactions between the microstructure and the dissipative deformation mechanisms in steels under cyclic loading", International Journal of Plasticity, vol. 32-33, pp. 106-120, 2012, doi: 10.1016/j.ijplas.2011.12.004.

[18] R. Peierls, "The size of a dislocation", Proceedings of the Physical Society, vol. 52, no. 1, p. 34, 1940.

[19] F. Nabarro, "Dislocations in a simple cubic lattice", Proceedings of the Physical Society, vol. 59, no. 2, p. 256, 1947.

[20] M. P. Luong, "Fatigue limit evaluation of metals using an infrared thermographic technique", Mechanics of materials, vol. 28, no. 1, pp. 155-163, 1998.

[21] F. Cura, G. Curti, and R. Sesana, "A new iteration method for the thermographic determination of fatigue limit in steels", International Journal of Fatigue, vol. 27, no. 4, pp. 453-459, 2005, doi: 10.1016/j.ijfatigue.2003.12.009.

[22] G. Fargione, A. Geraci, G. La Rosa, and A. Risitano, "Rapid determination of the fatigue curve by the thermographic method", International Journal of Fatigue, vol. 24, no. 1, pp. 11-19, 2002.

[23] R. De Finis, D. Palumbo, M. M. da Silva, and U. Galietti, "Is the temperature plateau of a selfheating test a robust parameter to investigate the fatigue limit of steels with thermography?", Fatigue \& Fracture of Engineering Materials \& Structures, vol. 41, no. 4, pp. 917-934, 2018, doi: $10.1111 /$ ffe.12738.

[24] C. Wang, A. Blanche, D. Wagner, A. Chrysochoos, and C. Bathias, "Dissipative and microstructural effects associated with fatigue crack initiation on an Armco iron", International Journal of Fatigue, vol. 58, pp. 152-157, 2014, doi: 10.1016/j.ijfatigue.2013.02.009.

[25] N. Connesson, F. Maquin, and F. Pierron, "Experimental energy balance during the first cycles of cyclically loaded specimens under the conventional yield stress", Experimental Mechanics, vol. 51, no. 1, pp. 23-44, 2010, doi: 10.1007/s11340-010-9336-4.

[26] L. Bodelot, L. Sabatier, E. Charkaluk, and P. Dufrénoy, "Experimental setup for fully coupled kinematic and thermal measurements at the microstructure scale of an AISI 316L steel", Materials Science and Engineering: A, vol. 501, no. 1-2, pp. 52-60, 2009, doi: 10.1016/j.msea.2008.09.053.

[27] N. Connesson, F. Maquin, and F. Pierron, "Dissipated energy measurements as a marker of microstructural evolution: 316L and DP600", Acta Materialia, vol. 59, no. 10, pp. 4100-4115, 2011, doi: 10.1016/j.actamat.2011.03.034.

[28] A. Robinson, J. Dulieu-Barton, S. Quinn, and R. Burguete, "Paint coating characterization for thermoelastic stress analysis of metallic materials", Measurement Science and Technology, vol. 21, no. 8, p. 085502, 2010.

[29] H. Louche and A. Chrysochoos, "Thermal and dissipative effects accompanying Lüders band propagation", Materials Science and Engineering: A, vol. 307, no. 1, pp. 15-22, 2001.

[30] P. Jaya Seelan, "Revealing the heterogeneity in weld microstructures using the thermomechanical dissipative heat source", PhD Thesis, University of Southampton, 2018. [Online]. Available: https://eprints.soton.ac.uk/427157/

[31] W. F. Hosford, Mechanical behavior of materials, First ed. Cambridge: Cambridge University Press, 2005.

[32] J. Schijve, Fatigue of structures and materials, First ed. USA: Springer, 2001.

[33] C. Donadille, R. Valle, P. Dervin, and R. Penelle, "Development of texture and microstructure during cold-rolling and annealing of FCC alloys: Example of an austenitic stainless steel", Acta metallurgica, vol. 37, no. 6, pp. 1547-1571, 1989. 
[34] G. J. Hopkin, "Modelling anisothermal recrystallization in austenitic stainless steels", PhD Thesis, University of Cambridge, 2002.

[35] P. Seelan, J. Dulieu-Barton, and F. Pierron, "The effect of microstructure on energy dissipation in 316L stainless steel", in Residual Stress, Thermomechanics \& Infrared Imaging, Hybrid Techniques and Inverse Problems, Volume 9: Springer, 2017, pp. 15-19.

[36] A. Benaarbia, A. Chrysochoos, and G. Robert, "Kinetics of stored and dissipated energies associated with cyclic loadings of dry polyamide 6.6 specimens", Polymer Testing, vol. 34, pp. 155-167, 2014, doi: 10.1016/j.polymertesting.2014.01.009.

[37] B. Yang et al., "Thermographic investigation of the fatigue behavior of reactor pressure vessel steels", Materials Science and Engineering A, vol. 314, pp. 131-139, 2001, doi: 10.1016/S0921-5093(00)01910-9.

[38] M. S. Pham, S. R. Holdsworth, K. G. F. Janssens, and E. Mazza, "Cyclic deformation response of AISI 316L at room temperature: mechanical behaviour, microstructural evolution, physically-based evolutionary constitutive modelling", International Journal of Plasticity, vol. 47, pp. 143-164, 2013, doi: 10.1016/j.ijplas.2013.01.017. 


\section{Figures}

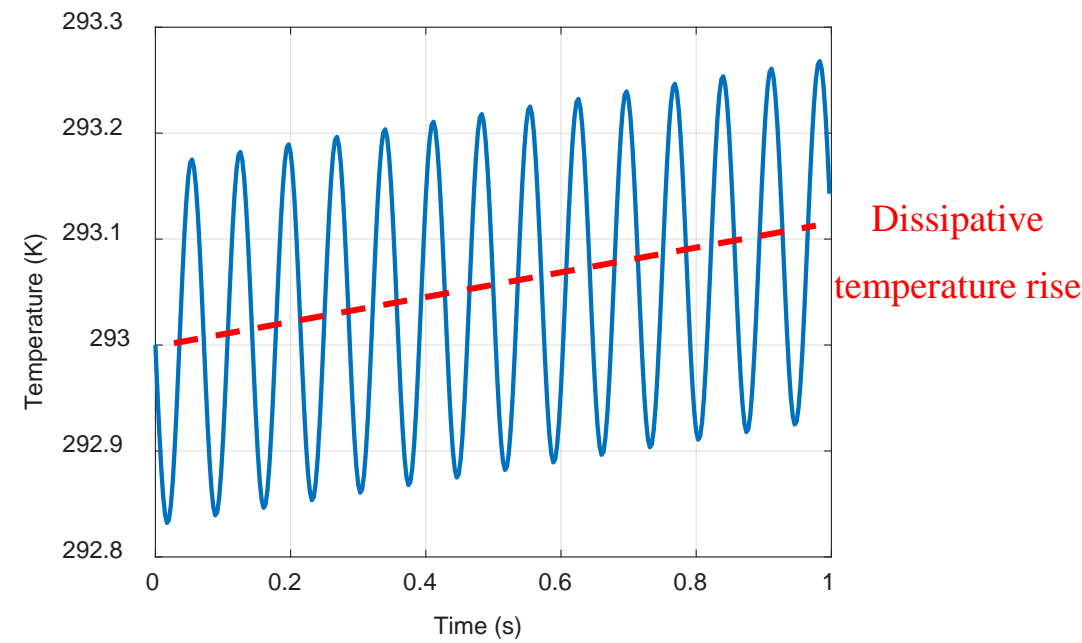

Figure 1 A schematic representation of temperature variation experienced in the first few cycles of elastic cyclic loading where the cyclic temperaure variation is superimposed on an increasing dissipative temperature rise. 


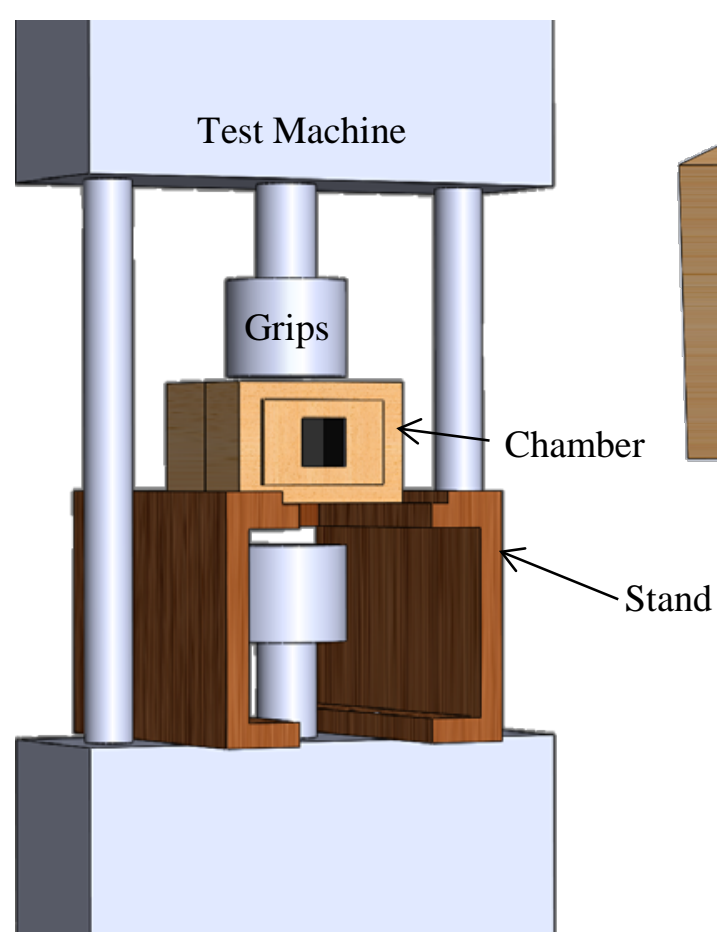

(a)

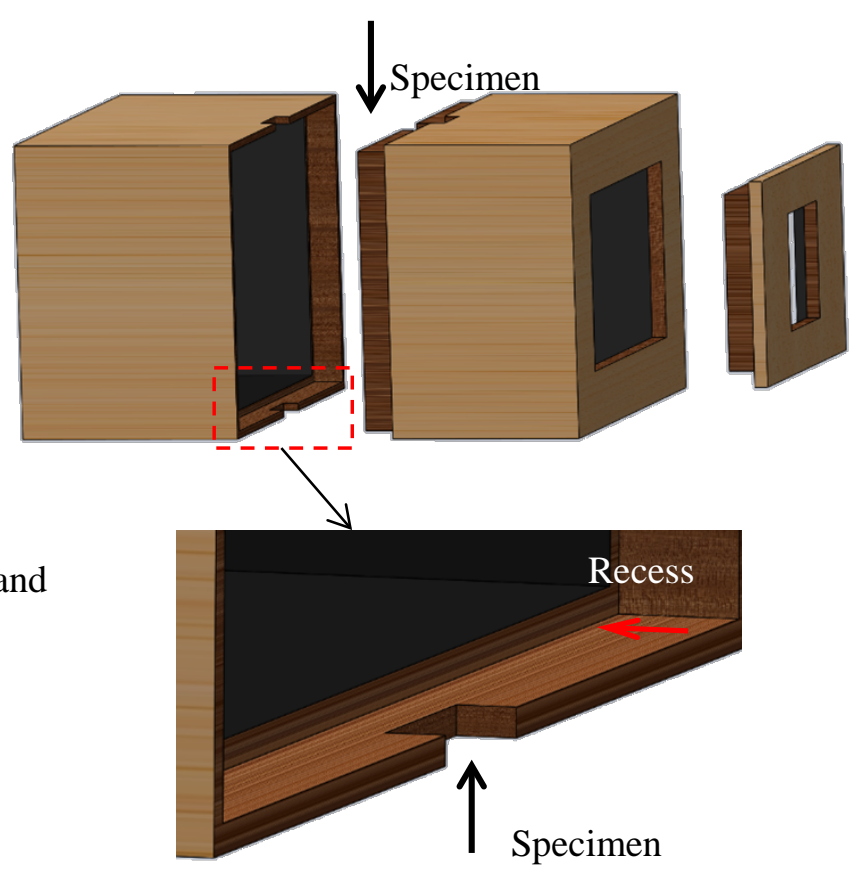

(b)

Figure 2 (a) Chamber in situ with the test machine (b) Exploded view of the chamber showing the different parts and features of the chamber. 


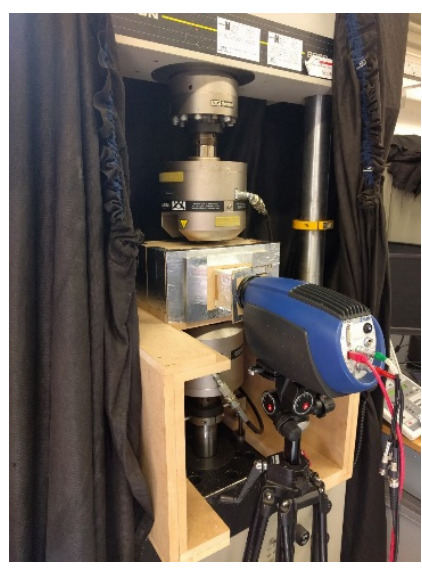

(a)

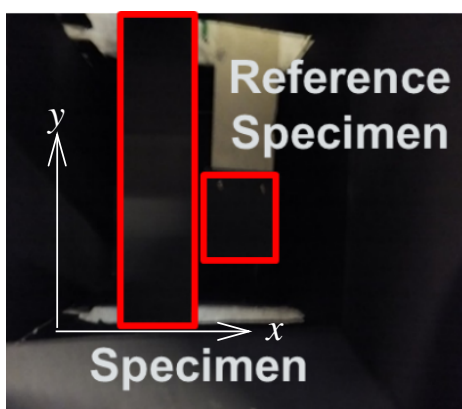

(b)

Figure 3 (a) Actual setup of the experiment (b) The position of the specimen and reference specimen within the chamber. 

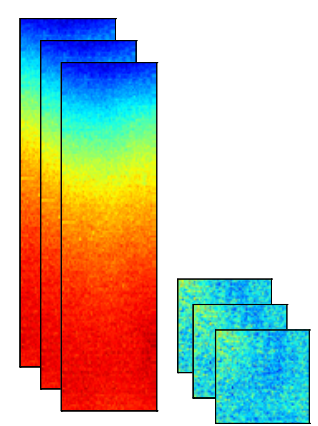

S

$\mathrm{R}$

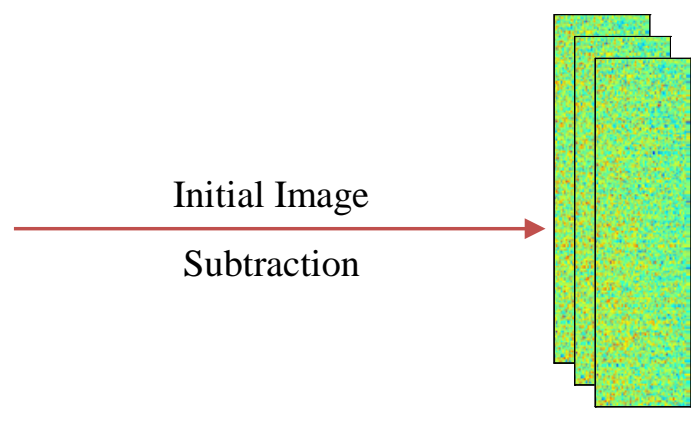

$\mathrm{S}$

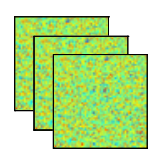

$\mathrm{R}$

(a)

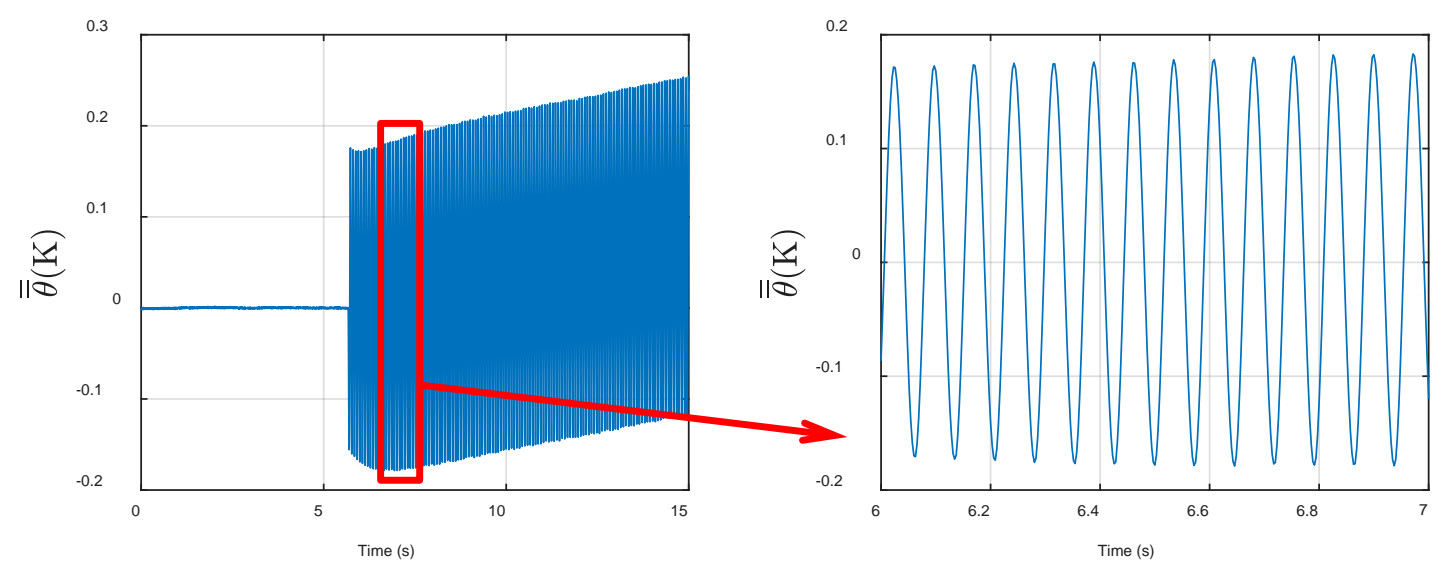

(b)

Figure 4 Data Processing Procedure: (a) Image Subtraction (b) Spatially averaged temperature variation 


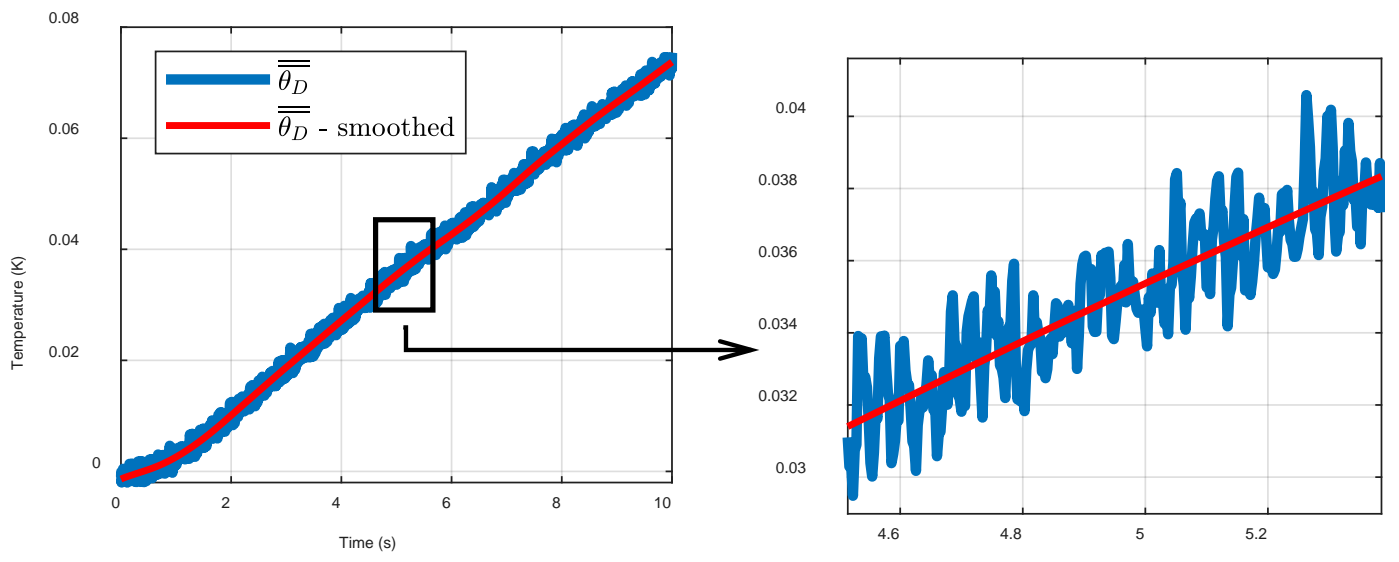

Figure 5 Mean temperature rise 


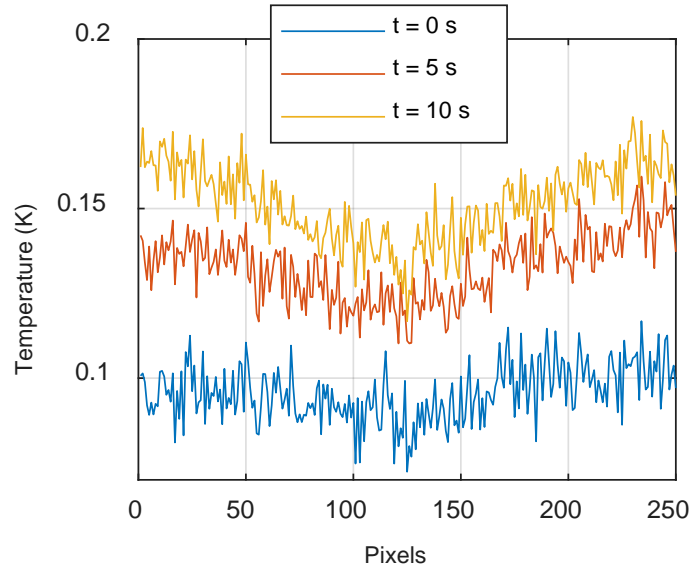

Figure 6 The evolution of the vertical temperature profile of the specimen during a test 


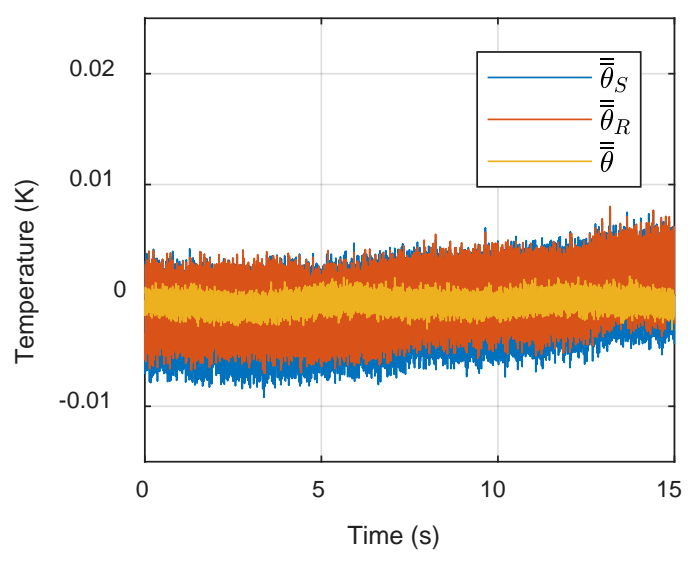

(a)

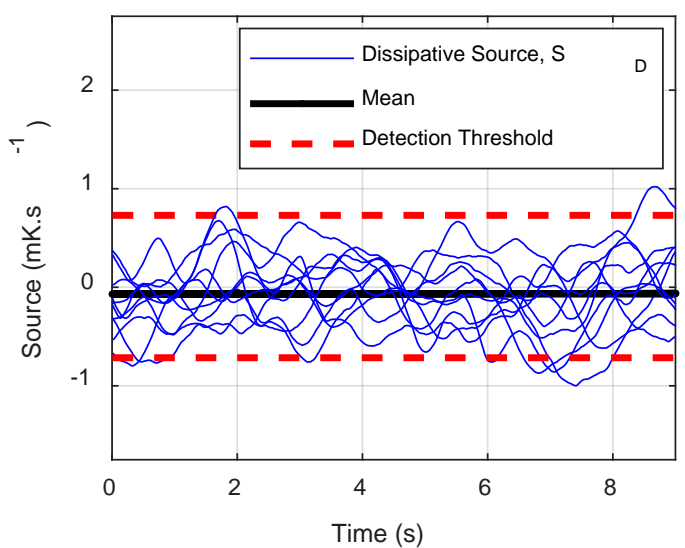

(b)

Figure 7 (a) Temperature variations and (b) the detection threshold for the dissipative source with the experimental set-up. 


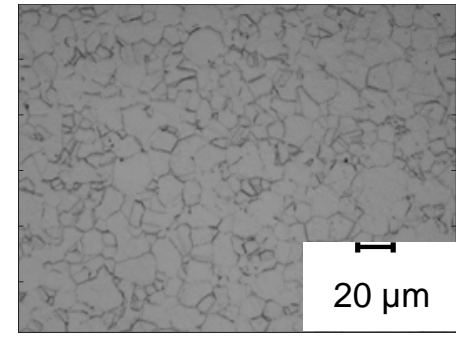

(a) Specimen A - as received

$$
20.0 \pm 1.8 \mu \mathrm{m}
$$

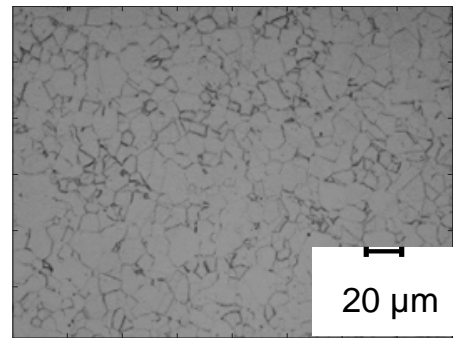

(d) Specimen D - $900{ }^{\circ} \mathrm{C}$

$$
19.4 \pm 4.3 \mu \mathrm{m}
$$

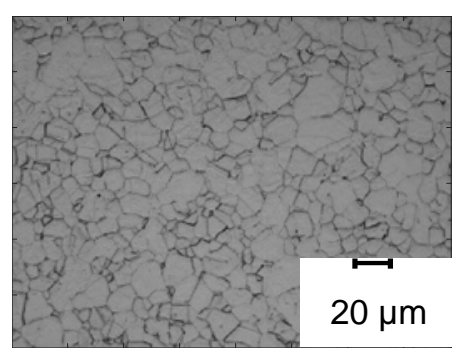

(b) Specimen $\mathrm{B}-450^{\circ} \mathrm{C}$ $21.2 \pm 5.1 \mu \mathrm{m}$

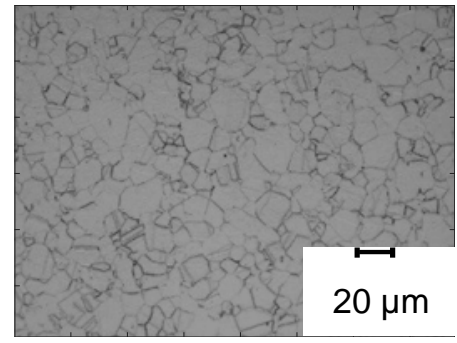

(c) Specimen $\mathrm{C}-650^{\circ} \mathrm{C}$ $21.3 \pm 5.4 \mu \mathrm{m}$

Figure 8 Microstructure of the heat treated specimens: (a) Specimen A, (b) Specimen B, (c) Specimen $C$, (d) Specimen $D,(e)$ Specimen $E$, together with average grain size 


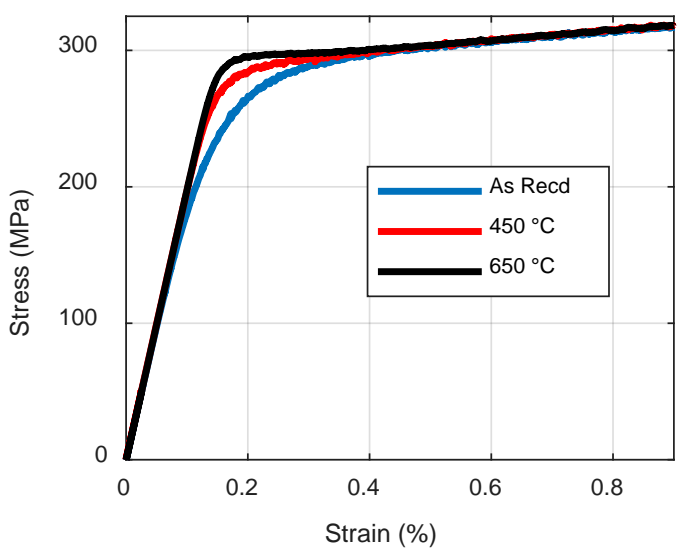

(a)

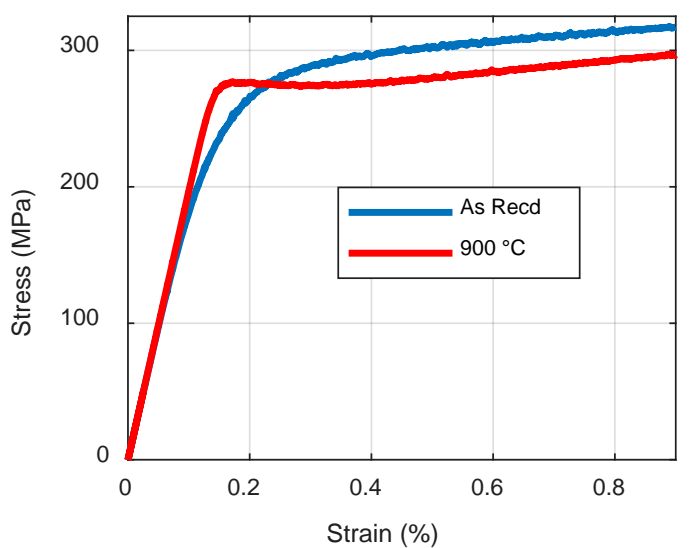

(b)

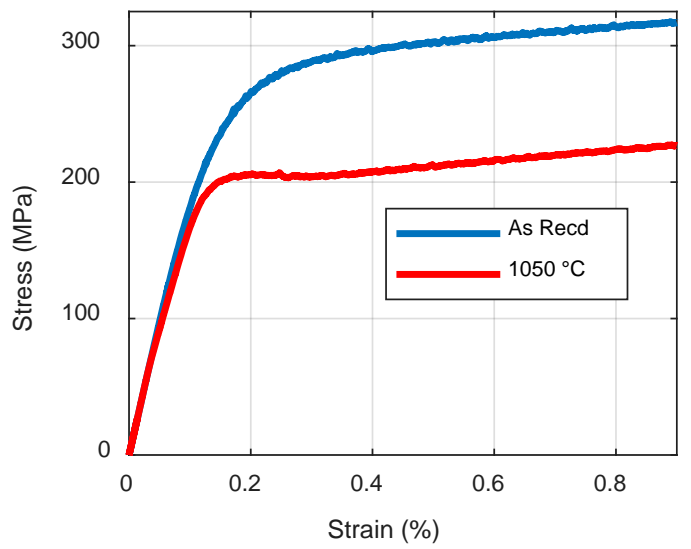

(c)

Figure 9 Stress strain curves of the heat treated specimens compared against the as received specimen: (a) Specimens $A, B \& C$, (b) Specimens $A$ and $D$, (c) Specimens $A$ and $E$ 
Table 1 Summary of Mechanical Properties

\begin{tabular}{cccccc}
\hline Specimen & $\begin{array}{c}\text { Temperature } \\
\left({ }^{\circ} \mathbf{C}\right)\end{array}$ & $\begin{array}{c}\text { Dwell Time } \\
\text { (hours) }\end{array}$ & $\begin{array}{c}\text { 0.2\% Offset Yield } \\
\text { Stress, } \boldsymbol{\sigma}_{\mathbf{y}} \text { (MPa) }\end{array}$ & $\begin{array}{c}\text { Hardness } \\
(\mathbf{H V})\end{array}$ & $\begin{array}{c}\text { Standard } \\
\text { Deviation (HV) }\end{array}$ \\
\hline A & As Received & - & 293 & 178 & 3.1 \\
\hline B & $450^{\circ} \mathrm{C}$ & 1 & 298 & 176 & 1.5 \\
$\mathbf{C}$ & $650^{\circ} \mathrm{C}$ & 1 & 299 & 176 & 5.1 \\
$\mathbf{D}$ & $900^{\circ} \mathrm{C}$ & 0.5 & 275 & 165 & 1.1 \\
$\mathbf{E}$ & $1050^{\circ} \mathrm{C}$ & 1 & 205 & 146 & 5.3 \\
\hline
\end{tabular}




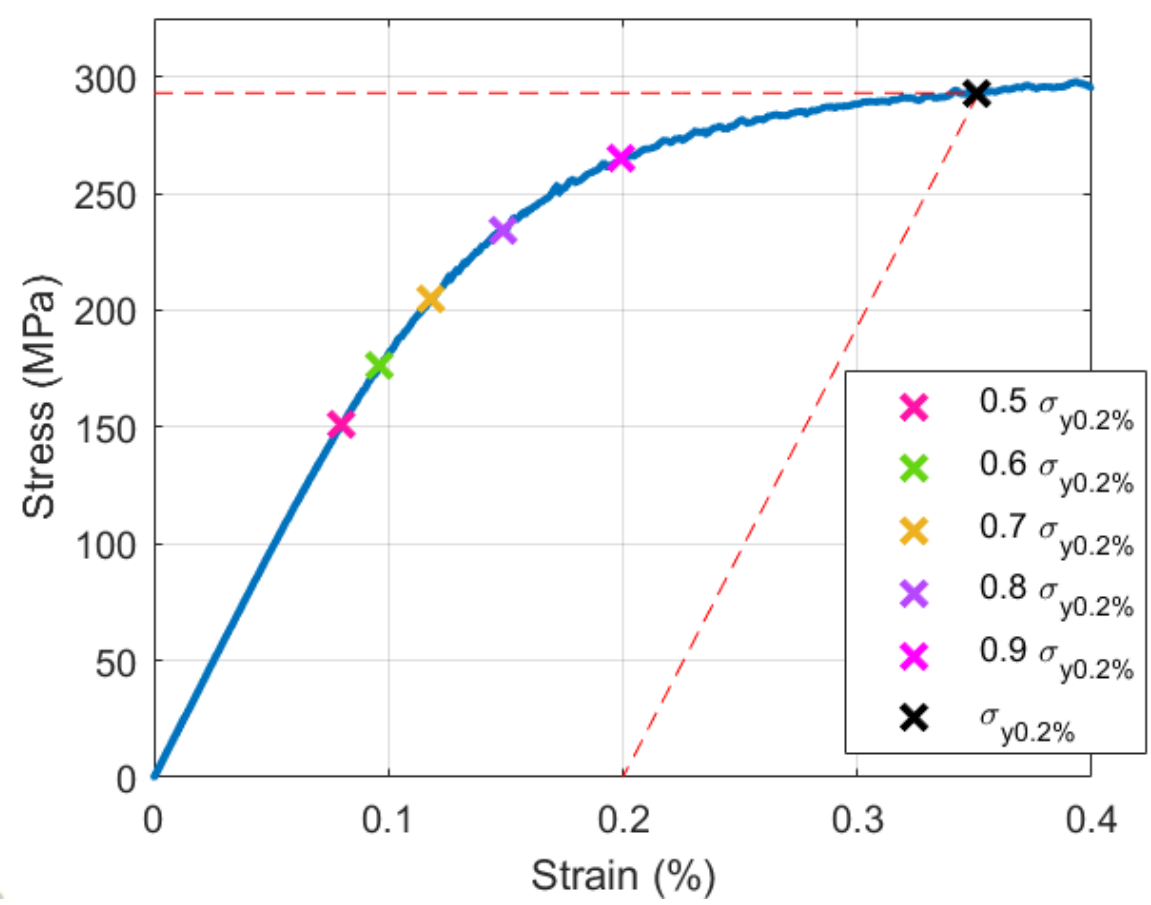

Figure 10 Maximum stress during the cyclic loading on the stress strain curve of the as received specimen 
Table 2 The sequence of applied loading for all test specimens

\begin{tabular}{ccc}
\hline Applied Maximum Stress, $\sigma_{\max }$ & \multicolumn{2}{c}{ Test Sequence } \\
\hline $\mathbf{0 . 5} \sigma_{\boldsymbol{y}}$ & 1 & 8 \\
$\mathbf{0 . 6} \sigma_{\boldsymbol{y}}$ & 2 & 9 \\
$\mathbf{0 . 7} \sigma_{y}$ & 3 & 10 \\
$\mathbf{0 . 8} \sigma_{y}$ & 4 & 11 \\
$\mathbf{0 . 9} \sigma_{\boldsymbol{y}}$ & 5 & 12 \\
$\mathbf{1 . 0} \sigma_{y}$ & 6 & 13 \\
$\approx \mathbf{1 . 2} \sigma_{\boldsymbol{y}}$ & 7 & \\
\hline
\end{tabular}




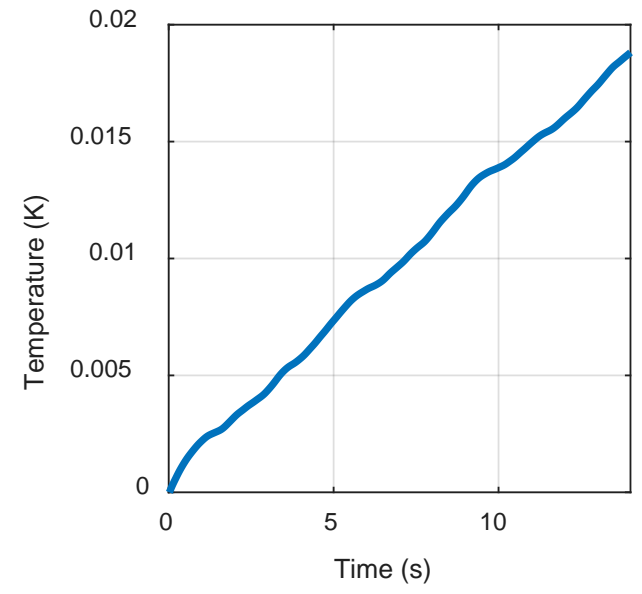

(a)

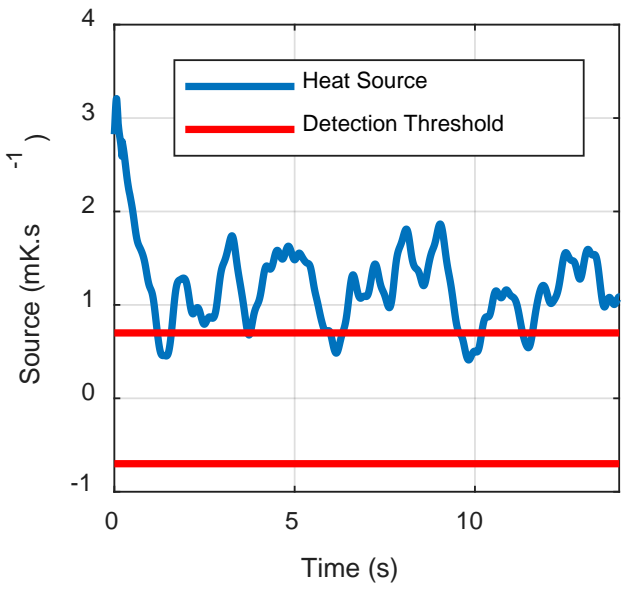

(b)

Figure 11 (a) Temperature rise (b) Corresponding heat source shown to be above the detection threshold under fully elastic cyclic loading (Specimen A - $\sigma_{\max }=205 \mathrm{MPa}$ ) 


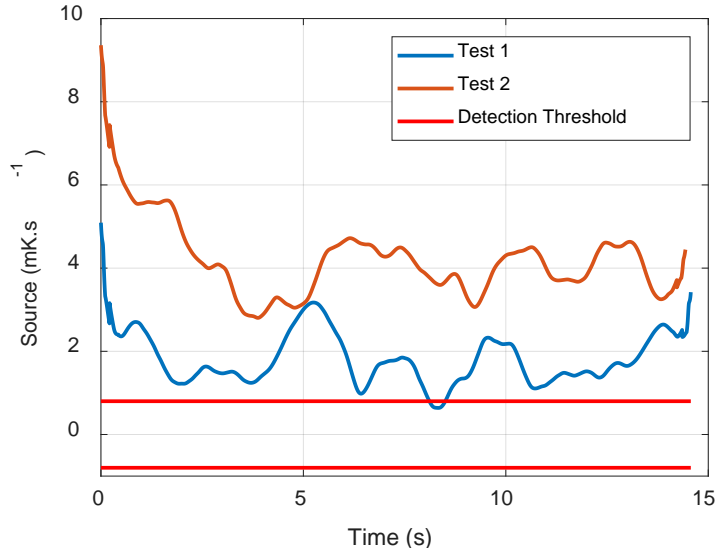

(a)

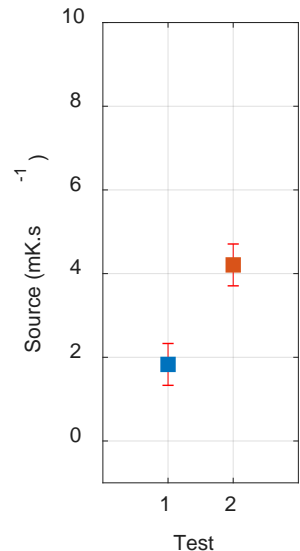

(b)

Figure 12 (a) Stabilised heat sources from fully elastic loading (b) Temporal average of (a) 


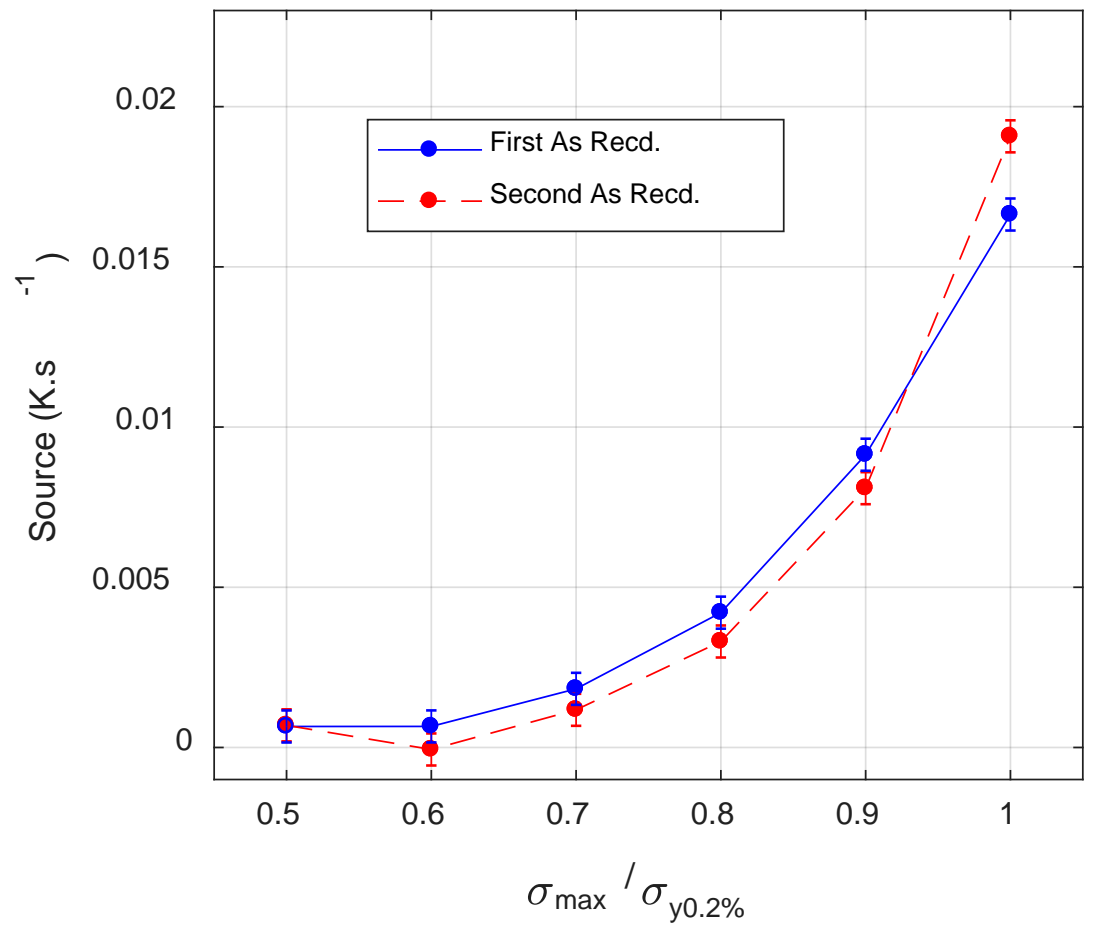

Figure 13 Comparison of the energy dissipated by two different specimens in the as received condition 


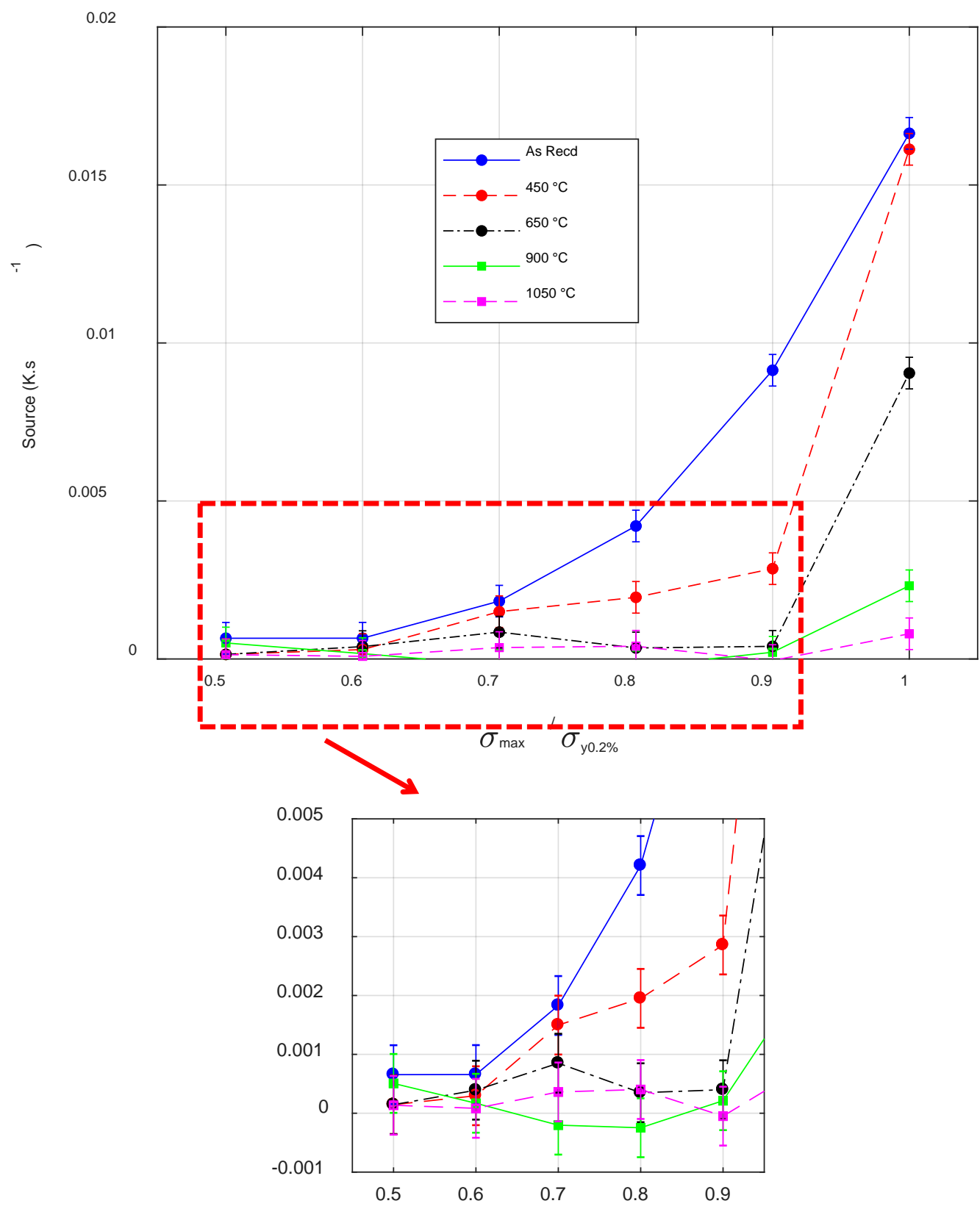

Figure 14 Energy dissipated at increasing stress levels below the macroscopic yield stress for specimens $A, B, C, D$ and $E$ 


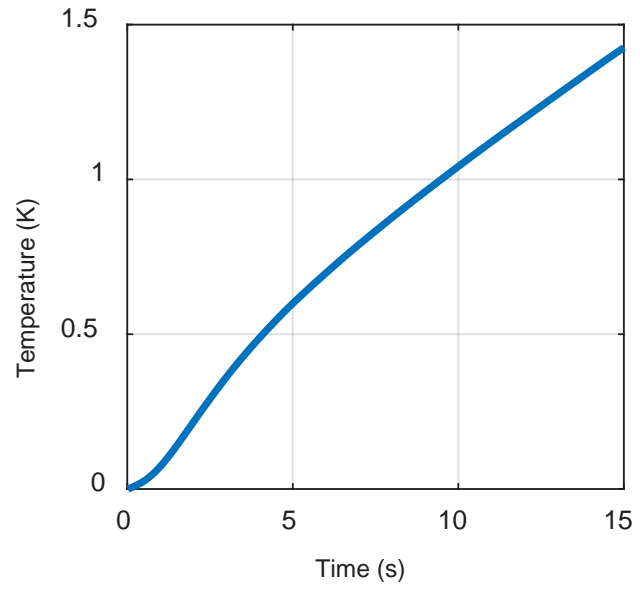

(a)

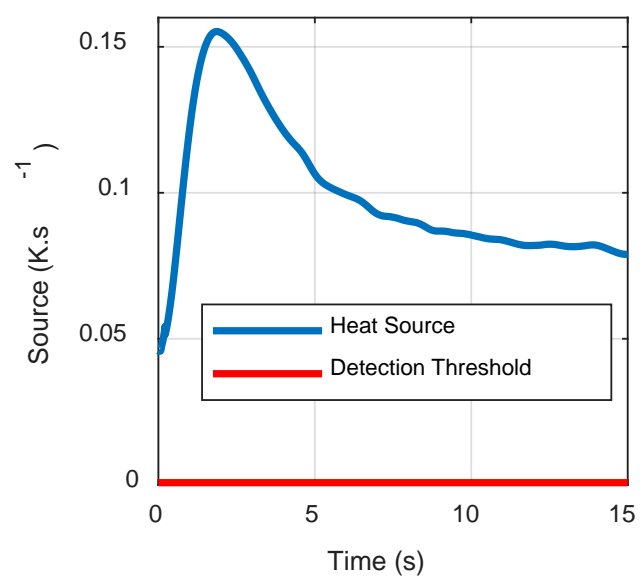

(b)

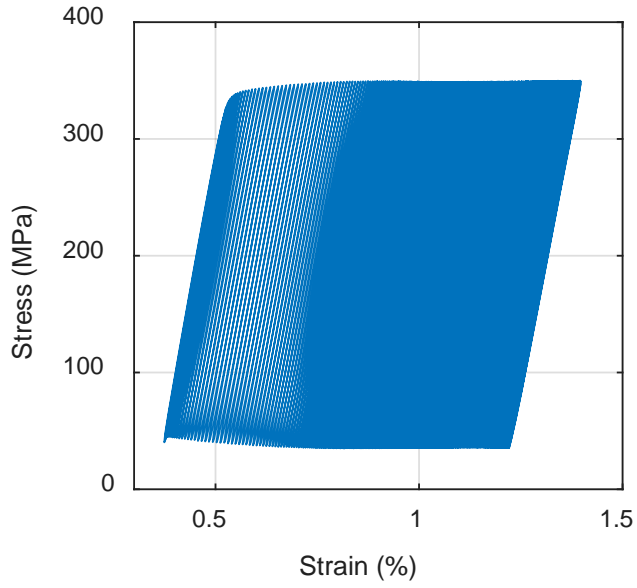

(c)

Figure 15 (a) Temperature rise (b) Corresponding heat source under elastic plastic cyclic loading (Specimen A - $\sigma_{\max }=350 \mathrm{MPa}$ ) (c) Simultaneously recorded stress strain curve of the elastic plastic loading (Specimen $A-\sigma_{\max }=350 \mathrm{MPa}$ ) 


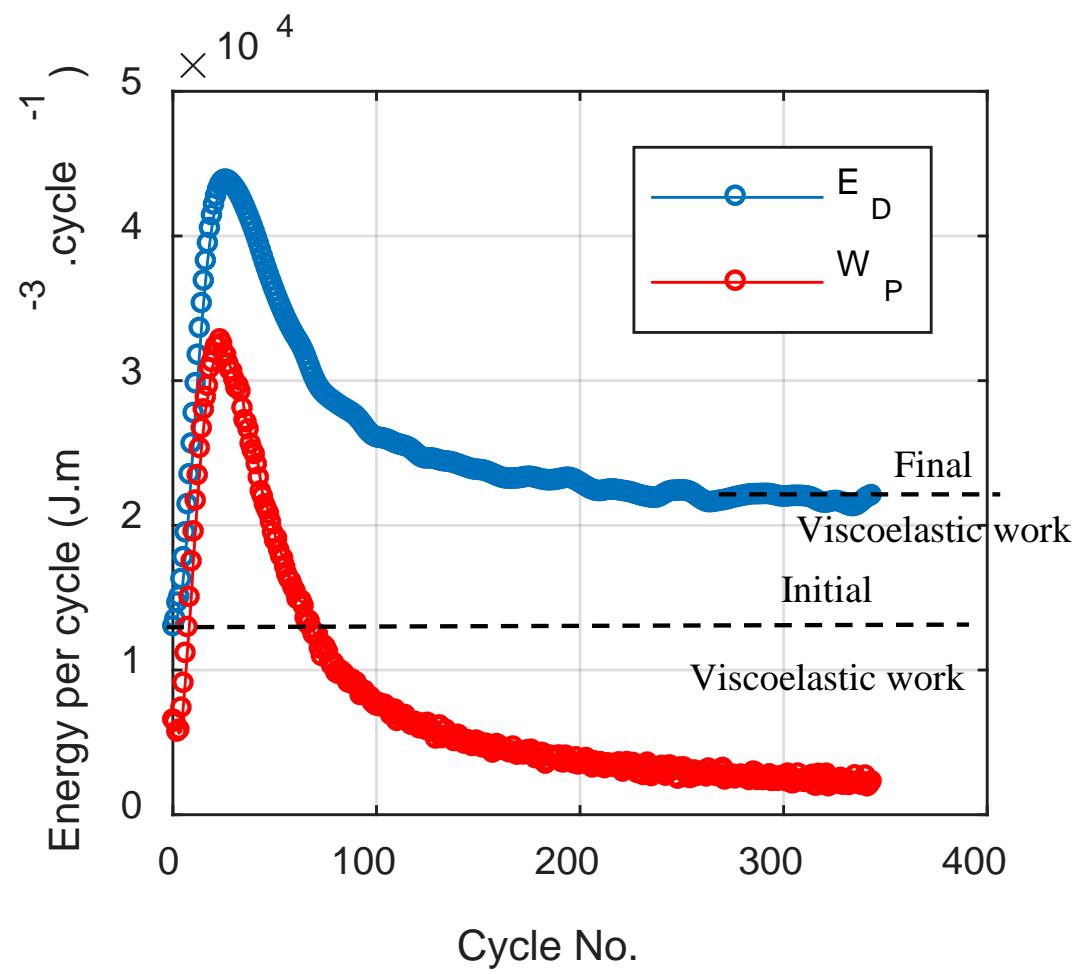

Figure 16 Comparison of energy dissipated and plastic work 


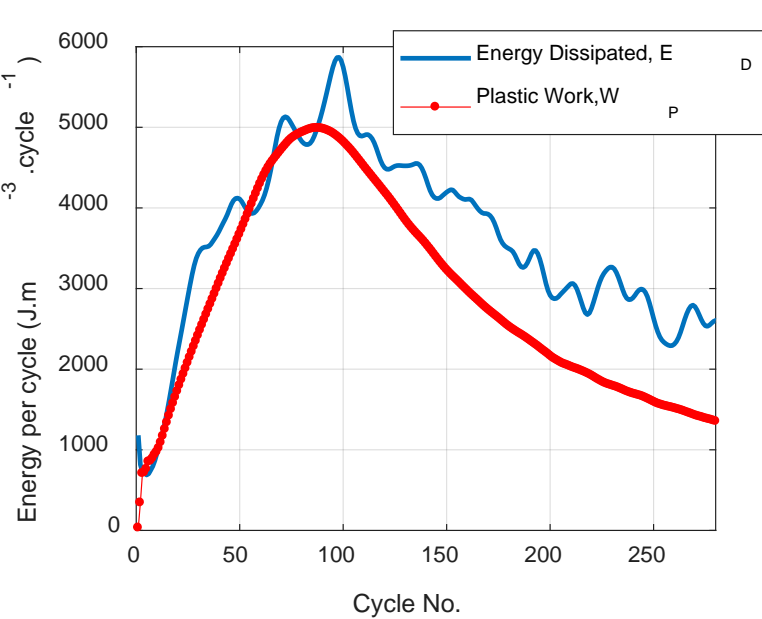

(a)

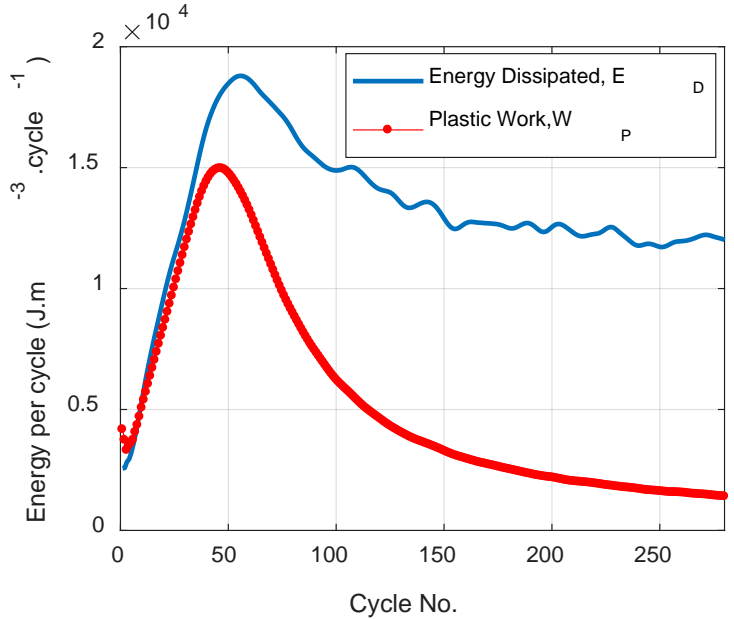

(b)

Figure 17 Comparison of plastic work to dissipated energy: (a) grain growth (specimen E) and (b) recrystallized (specimen D) 


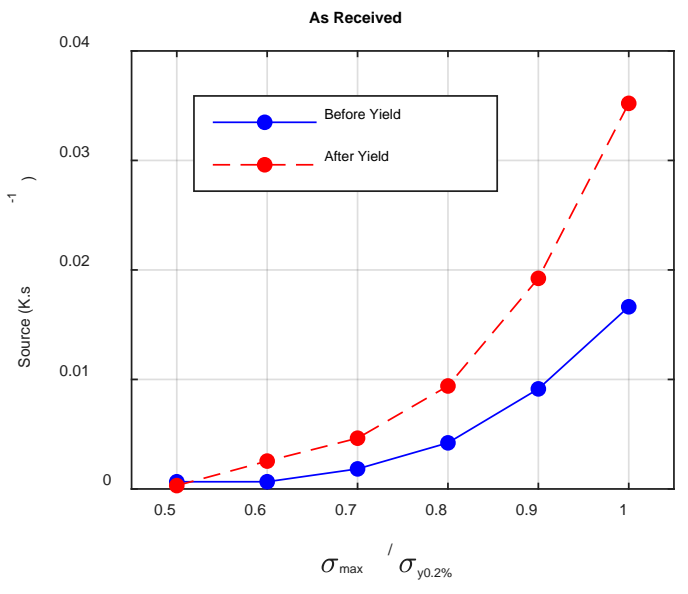

(a)

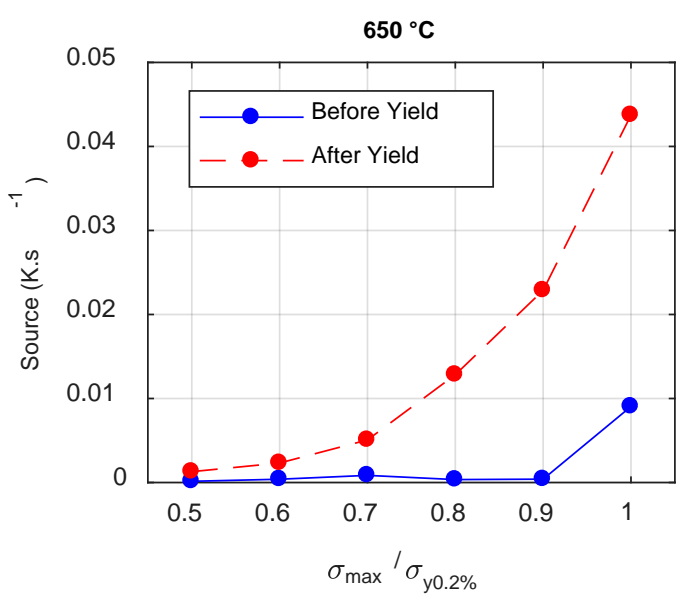

(c)

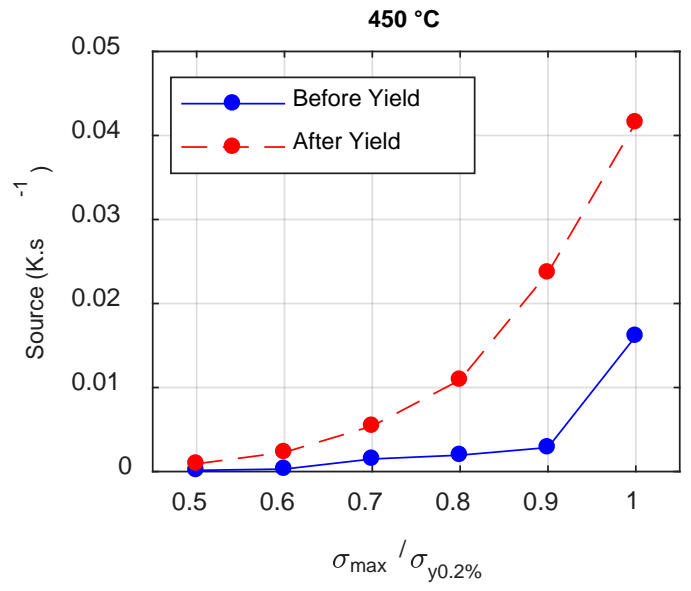

(b)

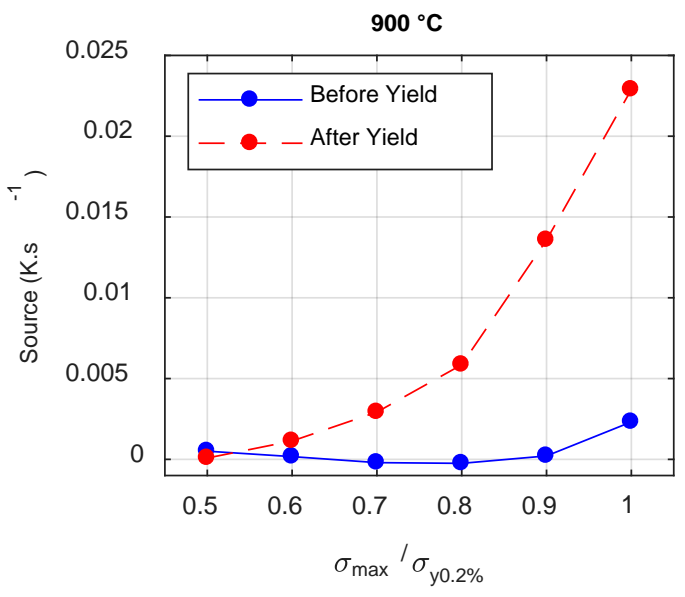

(d)

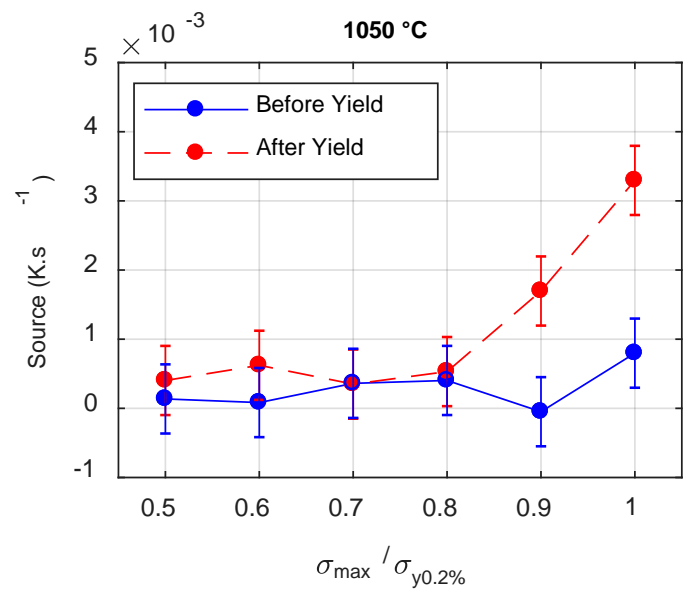

(e) 
Figure 18 Comparison between the energy dissipated before and after yielding for: (a) Specimen A - as received, (b) Specimen $B-450^{\circ} \mathrm{C}$, (c) Specimen $C$ - $650^{\circ} \mathrm{C}$, (d) Specimen $D-900{ }^{\circ} \mathrm{C}$, (e) Specimen $\mathrm{E}-1050^{\circ} \mathrm{C}$ 